\title{
General equilibrium in economies with uncertain delivery
}

\author{
João Correia-da-Silva ${ }^{1}$ and Carlos Hervés-Beloso ${ }^{2}$
}

May $15^{\text {th }}, 2011$.

\begin{abstract}
We consider a general equilibrium model of trade ex ante with differential information in which agents choose plans of state-contingent lists of bundles. Being unable to verify that the state of nature is $s$ and not $t$, an agent has to accept the delivery of any bundle in the list for delivery in state $s$ or in the list for delivery in state $t$. Under the assumption that each state of nature can be verified by at least one agent, we establish existence of equilibrium and we show that the equilibrium allocation satisfies a notion of coalitional incentive compatibility.
\end{abstract}

Keywords: General equilibrium, Differential information, Verifiability, Uncertain delivery, Lists of bundles, Rational behavior.

JEL Classification Numbers: C62, D51, D82.

\footnotetext{
* We are grateful to Ali Khan, Ana Paula Serra, Antonio Cabrales, Belén Jerez, David Levine, Diego Moreno, Emma Moreno, Enrico Minelli, Francesco de Sinopoli, Martin Meier, an anonymous referee and Nicholas Yannelis (the Editor), for useful comments and suggestions. João Correia-da-Silva (joao@fep.up.pt) acknowledges support from CEF.UP and research grant PTDC/EGEECO/111811/2009 from Fundação para a Ciência e Tecnologia and FEDER. Carlos Hervés-Beloso (cherves@uvigo.es) acknowledges support by research grants 10PXIB300141PR (Xunta de Galicia) and ECO2009-14457-C0401ECON (Ministerio de Educación y Ciencia).
}

\footnotetext{
${ }^{1}$ CEF.UP and Faculdade de Economia, Universidade do Porto, Portugal.

${ }^{2}$ RGEA, Facultad de Económicas, Universidad de Vigo, Spain.
} 


\section{Introduction}

In chapter 7 of his "Theory of Value", Debreu (1959) showed how to extend the general equilibrium model to the case of trade under uncertainty with public state verification. All that is needed is to consider a generalized notion of commodity that also includes in its description the state of nature on which its delivery is contingent (Arrow, 1953). The model becomes equivalent to the model without uncertainty (Arrow and Debreu, 1954; McKenzie, 1954): prices of the contingent commodities are announced, and agents choose the consumption plan that they prefer (specifying a consumption bundle for each of the possible states of nature), among those that satisfy their budget restriction; after trade agreements are made, the state of nature is publicly announced and agents receive the consumption bundle that corresponds to the announced state.

We are interested in studying the implications of differential information, in the form of private and incomplete state verification. While keeping the basic structure of the model, we assume that each agent is only able to verify (in a court of law, for contracts to be enforced) that the state of nature belongs to a set of his/her information partition.

The consequence of incomplete verification is that if an agent has bought different bundles for delivery in two states and is not able to verify whether the true state is one or the other, then he/she has to accept delivery of any of the two bundles. This is a natural generalization of the classical model, in which state verification is complete. ${ }^{3}$

To study this economic setting, we consider that objects of choice are plans of lists of bundles such that the agents have the right to receive one of the bundles in the list that corresponds to the state of nature that occurs (they have to accept any of the alternatives in the list). ${ }^{4}$ Contracts in which lists are traded are frequent.

A plane ticket gives you the right to travel if the plane is available at the date of departure, and, if the plane is not available, the right to stay in a hotel and travel on the next plane. But you cannot verify whether the plane is available or not. If, at the date of traveling, the airline announces that the plane is not available, you may have no alternative other than to accept staying in a hotel and traveling on the next day.

\footnotetext{
${ }^{3}$ A closely related line of research, initiated by Radner (1968), is based on the idea that the consequence of incomplete information is that an agent must consume the same in states of nature that he/she cannot distinguish.

${ }^{4}$ This concept builds on Arrow's (1953) notion of contingent goods. A contingent bundle is obviously a contingent list of bundles with a single element.
} 
Some car insurance contracts give you the right to use another car temporarily, in case of accident or malfunction. But the substitute car is left undefined in the contract. It is only stipulated that the car should belong to a certain class. It may be red or yellow, have radio or not, etc.

Suppose that agent $i$ cannot verify whether the state of nature is $s$ or $t$, but has, however, bought $x_{i}^{s}$ for delivery in state $s$ and $x_{i}^{t}$ for delivery in state $t$. Then: if state $s$ occurs, agent $i$ can receive $x_{i}^{s}$ or $x_{i}^{t}$. When receiving $x_{i}^{t}$ in state $s$, the agent cannot prove in a court of law that the contract has been violated (state $t$ could be the actual state and $x_{i}^{t}$ the contracted delivery). For the same reason: if state $t$ occurs, the possible deliveries are also $x_{i}^{s}$ or $x_{i}^{t}$. Observe that the set of alternatives that may be delivered, $\left\{x_{i}^{s}, x_{i}^{t}\right\}$, is the same in the set of states that the agent cannot distinguish, $\{s, t\}$.

Something that is constant across states of nature that agent $i$ is unable to distinguish is said to be "measurable with respect to private information" or $P_{i}$-measurable, where $P_{i}$ denotes the information partition of agent $i$. We can restrict our attention to $P_{i}$-measurable plans of lists, because, as exemplified above, any non-measurable choice can be converted into a measurable one that is equivalent. Buying a non-measurable consumption plan ( $x_{i}^{s}$ for state $s$ and $x_{i}^{t}$ for state $t)$, agent $i$ obtains a $P_{i}$-measurable plan of lists $\left(\left\{x_{i}^{s}, x_{i}^{t}\right\}\right.$ in state $s$ and $\left\{x_{i}^{s}, x_{i}^{t}\right\}$ in state $t$ ). It is important to understand that this $P_{i}$-measurability property of lists is not a restriction on trade, but the consequence of incomplete state verification on the enforceability of trade agreements.

This paper is a contribution to the theory of general equilibrium with differential information. We improve on the model of Radner (1968), essentially by considering that objects of choice are plans of contingent lists instead of plans of contingent bundles. The seminal work of Radner (1968) has been complemented by many developments. ${ }^{5}$ It is an open question whether these can be extended to the model presented here.

We have introduced this model of general equilibrium with private and incomplete state verification in two previous papers $(2008,2009)$. All trade is agreed ex ante, that is, before private information is received. Prices are announced, and agents choose the plan of contingent lists that they prefer, among those that belong to their budget set. After receiving their private

\footnotetext{
${ }^{5}$ Such as the private core notion (Yannelis, 1991), core-convergence results (Einy, Moreno and Shitovitz, 2001; Angeloni and Martins-da-Rocha, 2009), study of incentive compatibility (Koutsougeras and Yannelis, 1993; Krasa and Yannelis, 1994; Glycopantis, Muir and Yannelis, 2003), extension to infinite commodity spaces (HervésBeloso, Moreno-García and Yannelis, 2005; Podczeck and Yannelis, 2008) and infinite state spaces (Hervés-Beloso, Martins-da-Rocha and Monteiro, 2009), etc. For a comprehensive view, see the volume edited by Glycopantis and Yannelis (2005).
} 
information, agents are able to verify to which set of their information partition belongs the true state of nature. Then, each agent receives a bundle that belongs to the contingent list that corresponds to the actual state of nature, or to a state of nature that belongs to the same set of his/her information partition (the agent cannot prove that the contract has been violated). And, of course, the deliveries to all the agents in the economy must constitute a feasible allocation.

When buying a list, which of the alternatives should an agent expect to receive? In our preliminary explorations of this framework, we have studied the case of extreme pessimism and the case of continuous expectations. If agents expect to receive the worst possible bundle in a list, there exists an equilibrium in which these expectations are fulfilled. This is a prudent expectations equilibrium (2009). Agents act very defensively, selecting alternatives with the same utility for delivery in states that they cannot distinguish. They insure themselves completely against being deceived. Even if they are deceived, it implies no utility loss. A more general notion is that of a subjective expectations equilibrium (2008), where the agent's beliefs about the probabilities of delivery of the different alternatives in a list are a continuous function of the prices that they observe (perfectly or imperfectly) and of the alternatives specified in the list.

In this paper, we study the case of rational behaviour: agents know the model of the economy, and form their expectations accordingly (Muth, 1961). ${ }^{6}$ This solution concept is more consistent, but also more challenging to investigate. In the case of prudent or subjective expectations, equilibrium could be established by more or less direct application of Berge's Maximum theorem and Kakutani's fixed point theorem. Now, with agents having rational expectations, to establish existence of an equilibrium we construct a sequence of Arrow-Debreu economies and show that the limit of the sequence equilibria is an equilibrium of our economy.

In our model: (1) the price of a plan of contingent lists (specifying a list of possible bundles for delivery in each state of nature) is equal to the price of the cheapest consumption plan (specifying a bundle for delivery in each state of nature) that satisfies the requirements of the plan of contingent lists; and (2) this cheapest consumption plan is actually the alternative that is selected for delivery (in each state, the bundle that is selected for delivery is the cheapest according to prices for delivery in this state).

\footnotetext{
${ }^{6} \mathrm{~A}$ different line of research, associated with the notion of rational expectations, focuses on the revelation of information by prices (Radner, 1979; Allen, 1981). But with trade taking place ex ante, an agent cannot infer the information of the other agents because, at the moment of trade, the other agents still haven't received their information. From the deliveries made at date 1, agents could be able to infer the true state of nature. But we assume that the information obtained through these inferences cannot be used (in a court of law, for example) to enforce contracts.
} 
Rational agents expect, then, to receive the cheapest possible alternative in each state of nature. ${ }^{7}$ Observing the prices of all the contingent commodities and of all the lists, they can predict which bundle is going to be selected for delivery in each state of nature. In case of a tie, agents expect to receive the alternative that they prefer (a similar assumption is made in the mechanism design literature: in case of indifference, agents are truthful).

Being able to anticipate the consumption plan that results from buying each plan of contingent lists, agents can, instead of choosing a plan of lists, choose the resulting consumption plan. Deliverable consumption plans are those that satisfy a system of linear inequalities. Consider an agent who does not distinguish between states $s$ and $t$. For a consumption plan, $\left(x_{i}^{s}, x_{i}^{t}\right)$, to be deliverable, it must be such that $p^{s} \cdot x_{i}^{s} \leq p^{s} \cdot x_{i}^{t}$ and $p^{t} \cdot x_{i}^{t} \leq p^{t} \cdot x_{i}^{s}$. If these deliverability conditions are not satisfied, then the agent will not receive $x_{i}^{s}$ in state $s$ and $x_{i}^{t}$ in state $t$ (because these would not be the cheapest alternatives in the corresponding states). An agent with rational expectations chooses among plans which are deliverable in this sense (denoted $x_{i} \in C_{i}(p)$ ).

This deliverable choice set depends, therefore, on prices and on each agent's private information. The choice set of each agent is the intersection of the budget set and the deliverable set, $B_{i}(p) \cap$ $C_{i}(p)$. If the correspondence from prices to the choice set were continuous, equilibrium existence would be guaranteed. In a bounded economy, $B_{i}(p) \cap C_{i}(p)$ is upper hemicontinuous. But $C_{i}(p)$ is not lower hemicontinuous. ${ }^{8}$ This property fails when prices in some state are null or when prices in states $s$ and $t$, with $t \in P_{i}(s)$, are collinear. ${ }^{9}$

We give an example of non-existence of equilibrium caused by null prices. In the presence of differential information, prices for delivery (of any commodity) in some state may be null, even if state-contingent preferences are strictly monotonic. In such a state, resources are abundant, but no agent can verify that this state has occurred. As a result, no agent is willing to pay a positive price for delivery contingent on the occurrence of this state.

To establish existence of equilibrium, we assume that any state of nature can be verified by at least one agent. The strategy of the proof is to construct a sequence of Arrow-Debreu

\footnotetext{
${ }^{7}$ Prices differ across states, thus, the cheapest bundle may also differ (which implies that the consumption plan may not be $P_{i}$-measurable).

${ }^{8}$ The intersection of continuous correspondences may not be continuous, anyway (Aliprantis and Border, 2007).

${ }^{9}$ With agents having preferences that are $P_{i}$-measurable, collinearity does not prevent existence of equilibrium. In this case, it can be shown that (having convex preferences) agents choose the same bundle for delivery in both states, implying that the deliverability restrictions are satisfied in equality.
} 
economies in which agents that violate the deliverability constraints suffer a utility penalty, which is increasingly severe along the sequence. This sequence of economies has a sequence of equilibria, whose limit is an equilibrium of our differential information economy. ${ }^{10}$

Under the same assumption, we show that the equilibrium allocation satisfies weak coalitional incentive compatibility in the sense of Koutsougeras and Yannelis (1993). After the contracting stage, if state $s$ occurs, a coalition of agents could be interested in misrepresenting its information, so that the net trades that were agreed for state $t$ would be carried out instead of those that were agreed for state $s$. We show that no coalition of agents who are able to verify that $s$ is the true state of nature is interested in sending false reports that do not contradict the supposedly truthful reports of the other agents.

The paper is organized as follows: in section 2, we present the basic setup, explain the consequences of incomplete information and describe prices and preferences over lists; in section 3, we define and characterize equilibrium; in section 4, we establish existence and coalitional incentive compatibility; and, in section 5, we make some concluding remarks. In appendix, we: (1) collect all the proofs, (2) give an example of non-existence of equilibrium, and (3) study continuity of the deliverability correspondence.

\section{The economy}

\subsection{Basic setup}

The economy extends over two time periods, date 0 and date 1 . There is a finite number of agents, $\mathcal{I}=\{1, \ldots, I\}$, who trade (at date 0) a finite number of commodities, $\mathcal{L}=\{1, \ldots, L\}$, under uncertainty about which of a finite number of possible states of nature, $\mathcal{S}=\{1, \ldots, S\}$, will occur (at date 1). The state of nature determines the endowments and preferences of the agents.

At date 0 , agents know the probabilities of occurrence of each state, $\mu=\left(\mu^{1}, \ldots, \mu^{S}\right) \in \Delta^{S}$. At date 1 , if state $s$ occurs, each agent is only able to verify (and prove in a court of law, for

\footnotetext{
${ }^{10}$ The assumption that any state of nature can be verified by at least one agent implies that the prices in the limit are strictly positive, and this allows us to show that they are actually the equilibrium prices. In the model of Radner (1968), if free disposal is not allowed, the same assumption is necessary to guarantee the existence of equilibrium with non-negative prices.
} 
contracts to be enforced) that the state of nature belongs to the corresponding set of his/her information partition, $P_{i}(s)$.

Knowing their $P_{i}$-measurable vector of strictly positive state-dependent endowments, $e_{i} \in \mathbb{R}_{++}^{S L} \cap$ $P_{i}$, agents make (at date 0) contingent trade agreements with the objective of obtaining a consumption plan, $x_{i} \in \mathbb{R}_{+}^{S L}$, that maximizes their expected utility, $U_{i}\left(x_{i}\right)=\sum_{s \in \mathcal{S}} \mu^{s} u_{i}^{s}\left(x_{i}^{s}\right)$, where $u_{i}^{t}=u_{i}^{s}, \forall t \in P_{i}(s)$. Afterwards (at date 1), agents receive their endowments and their private information about the state of nature, and trade agreements are carried out.

In the case of public state verification (Debreu, 1959), agents trade contingent commodities. At date 0 , taking as given the price system, $p \in \Delta^{S L}=\left\{p \in \mathbb{R}_{+}^{S L}: \sum_{s \in \mathcal{S}} \sum_{l \in \mathcal{L}} p^{s l}=1\right\}$, agents select the consumption plan that maximizes their expected utility, among those that belong to their budget set, $B_{i}(p)=\left\{x_{i} \in \mathbb{R}_{+}^{S L}: \sum_{s \in \mathcal{S}} p^{s} \cdot x_{i}^{s} \leq \sum_{s \in \mathcal{S}} p^{s} \cdot e_{i}^{s}\right\}$. At date 1 , the state of nature is publicly announced, and the corresponding trade agreements take place (if state $s$ occurs, each agent $i$ delivers his/her endowment, $e_{i}^{s}$, and receives the consumption bundle that he/she is entitled to, $\left.x_{i}^{s}\right)$.

What happens if agents receive different information? What happens if, instead of being publicly verifiable, the state of nature is only privately and incompletely verifiable by each of the agents?

To answer this question, we consider the model of an economy with uncertain delivery, in which agents select plans of lists instead of consumption plans. At date 0, taking as given the prices of plans of lists, $\tilde{p}$, each agent $i$ chooses the plan of lists that he/she prefers, $\tilde{x}_{i}$, among those that belong to his/her budget set. The plan of lists specifies a set of possible consumption bundles, $\tilde{x}_{i}^{s}$, for delivery in each state of nature, $s$. At date 1 , if state $s$ occurs, agent $i$ delivers his/her endowment, $e_{i}^{s}$, and receives one of the alternatives in the list $\tilde{x}_{i}^{s}$ (truthful delivery) or an alternative in another list, $\tilde{x}_{i}^{t}$, contracted for delivery in an undistinguishable state of nature, $t \in P_{i}(s)$ (concealed violation).

\subsection{The solution of Radner}

In a seminal contribution, Radner (1968) postulated that in states of nature that an agent does not distinguish, $s$ and $t$ such that $t \in P_{i}(s)$, the same bundle would be consumed. By simply restricting the consumption set to $\mathbb{R}_{+}^{S L} \cap P_{i}$ (meaning that if $t \in P_{i}(s)$, then $x_{i}^{t}=x_{i}^{s}$ ), the classical model could be reinterpreted to cover the case of private information. 
Before presenting a critique of this solution, and an alternative concept, we stress that, in our economy: having made a contract for the contingent delivery of commodities, an agent needs to prove that an event has occurred to enforce delivery. The meaning of the information partition, $P_{i}$, is that, if state $s$ occurs, agent $i$ can prove that the state of nature belongs to $P_{i}(s)$, and can use this and only this information to enforce delivery.

The main objection to the model of Radner (1968) is that agents should not be restricted to consume the same bundle in states of nature that they do not distinguish (even if contracts are only contingent upon events that they can observe). The example that follows shows that such restriction is too strong.

Consider an economy with two agents. Agent $A$ is endowed with two units of 'sugar', in all states of nature, $\mathcal{S}=\left\{s_{1}, s_{2}\right\}$, while agent $B$ has uncertain endowments: two units of 'tea' in state $s_{1}$ and two units of 'coffee' in state $s_{2}$ :

$$
e_{A}^{s_{1}}=e_{A}^{s_{2}}=(2,0,0), \quad e_{B}^{s_{1}}=(0,2,0) \text { and } e_{B}^{s_{2}}=(0,0,2)
$$

The preferences of the agents are the same, and do not depend on the state of nature. The goods 'tea' and 'coffee' are perfect substitutes, that agents like to drink with 'sugar':

$$
u_{A}^{s_{1}}=u_{A}^{s_{2}}=u_{B}^{s_{1}}=u_{B}^{s_{2}}=\sqrt{\left(x_{t e a}+x_{c o f}\right) x_{s u g}} .
$$

Agent $A$ cannot distinguish the two states, which are equiprobable:

$$
P_{A}=\left\{s_{1}, s_{2}\right\} \text { and } P_{B}=\left\{\left\{s_{1}\right\},\left\{s_{2}\right\}\right\}
$$

With the restriction of consuming the same in indistinguished states of nature, there is no trade. To see this, observe that agent $A$ would like to consume some 'tea' in state $s_{1}$. But this would imply equal consumption in state $s_{2}$, and there is no 'tea' in state $s_{2}$ (only 'coffee'...).

In a real-life situation, the two agents could make the following agreement (valid for both states of nature): agent $A$ would deliver one unit of 'sugar' in exchange for one unit of 'tea' or one unit of 'coffee'. Agent $A$ would get the right to receive 'tea or coffee', or, to put it another way, would get the right to consume $(1,1,0)$ or $(1,0,1)$. Both agents would end up consuming $(1,1,0)$ in state $s_{1}$ and $(1,0,1)$ in state $s_{2}$. This contract for uncertain delivery allows the agents to attain an optimal outcome. ${ }^{11}$

Agent $A$ is buying what we call a list of bundles: a derivative good that gives him/her the right

\footnotetext{
${ }^{11}$ For other examples and a more detailed explanation, see our previous work $(2008,2009)$.
} 
to receive one of the bundles in the list. This suggests that, to improve upon the solution of Radner (1968), we should allow agents to trade lists of bundles. Then, some questions arise:

(1) What are the consequences of private state verification?

(2) What is the price of a list of bundles?

(3) What is the utility of a list of bundles?

\subsection{The consequences of private state verification}

We do not restrict each agent $i$ to select $P_{i}$-measurable consumption plans. Agents are allowed to buy different rights for delivery in states that they do not distinguish. But, if an agent buys different rights for delivery in two states and is not able to verify whether the true state is one or the other, then the agent will have to accept delivery of any of the two.

As an example, consider an agent $i$ who has bought the bundle $x_{i}^{s}$ for delivery in state $s$ and the bundle $x_{i}^{t}$ for delivery in state $t$. However, the agent cannot prove in a court of law whether the true state of nature is $s$ or $t$. Therefore, when receiving the bundle $x_{i}^{t}$ in state $s$ (or the bundle $x_{i}^{s}$ in state $t$ ), the agent cannot prove that the contract is being violated. This means that: if state $s$ occurs, the agent can receive $x_{i}^{s}$ or $x_{i}^{t}$; and if state $t$ occurs, the agent can also receive either $x_{i}^{s}$ or $x_{i}^{t}$. Observe that the set of alternatives that may be delivered, $\left\{x_{i}^{s}, x_{i}^{t}\right\}$, is constant across states that the agent cannot distinguish, $\{s, t\}$.

The same reasoning applies to lists. Suppose that agent $i$ has bought the right to receive a bundle in the list $\tilde{x}_{i}^{s}$ if state $s$ occurs and a bundle in the list $\tilde{x}_{i}^{t}$ if state $t$ occurs. In state $s$, agent $i$ may receive $x_{i}^{s} \notin \tilde{x}_{i}^{s}$ (the bundle that is delivered in state $s, x_{i}^{s}$, may not belong to the list that was contracted for delivery in state $s, \tilde{x}_{i}^{s}$ ). The delivered bundle may belong to a list contracted for delivery in a state $t \in P_{i}(s)$ (this would be a concealed violation of the contract). All that the agent can guarantee is that the bundle delivered in state $s$ belongs to $\bigcup_{t \in P_{i}(s)} \tilde{x}_{i}^{t}$.

Notice that if agent $i$ buys the same lists for delivery in the states that he cannot distinguish,

then $\bigcup_{t \in P_{i}(s)} \tilde{x}_{i}^{t}=\tilde{x}_{i}^{s}$, and therefore the agent will receive what she bought: a bundle $x_{i}^{s} \in \tilde{x}_{i}^{s}$ (in state $s)$.

Formally, for each agent $i \in \mathcal{I}$ :

(i) A contingent list for delivery in state $s$ is a finite and non-empty subset of $\mathbb{R}_{+}^{L}$, denoted 
$\tilde{x}_{i}^{s} \in \mathbb{F}\left(\mathbb{R}_{+}^{L}\right) ;^{12}$

(ii) A plan of lists is a vector of contingent lists, $\tilde{x}_{i} \in\left(\mathbb{F}\left(\mathbb{R}_{+}^{L}\right)\right)^{S}$, specifying a list for delivery in each of the possible states of nature;

(iii) a $P_{i}$-measurable plan of lists is a vector of contingent lists such that $t \in P_{i}(s) \Rightarrow \tilde{x}_{i}^{t}=\tilde{x}_{i}^{s}$, denoted $\tilde{x}_{i} \in\left(\mathbb{F}\left(\mathbb{R}_{+}^{L}\right)\right)^{S} \cap P_{i}$.

To each plan of lists corresponds a list of consumption plans, $\Pi\left(\tilde{x}_{i}\right) \in \mathbb{F}\left(\mathbb{R}_{+}^{S L}\right)$, defined as the collection of all the consumption plans, $x_{i} \in \mathbb{R}_{+}^{S L}$, that can be formed by selecting, for each state of nature, a bundle of the corresponding contingent list, $x_{i}^{s} \in \tilde{x}_{i}^{s}$. It is defined, thus, as the cartesian product of the contingent lists: $\Pi\left(\tilde{x}_{i}\right)=\Pi_{s \in \mathcal{S}} \tilde{x}_{i}^{s}$.

We define a transformation, $M_{i}$, to describe the consequences of incomplete information. If agent $i$ buys a plan of lists $\tilde{x}_{i}$, the set of consumption bundles that he/she may receive in state $s$ is $M_{i}^{s}\left(\tilde{x}_{i}\right)$, defined as:

$$
\begin{aligned}
& M_{i}^{s}:\left(\mathbb{F}\left(\mathbb{R}_{+}^{L}\right)\right)^{S} \longrightarrow \mathbb{F}\left(\mathbb{R}_{+}^{L}\right) ; \\
& M_{i}^{s}\left(\tilde{x}_{i}\right)=\bigcup_{t \in P_{i}(s)} \tilde{x}_{i}^{t} .
\end{aligned}
$$

Therefore, for agent $i$, buying $\tilde{x}_{i}$ is equivalent, from the point of view of deliveries, to buying the $P_{i}$-measurable (by construction) plan of lists $M_{i}\left(\tilde{x}_{i}\right)=\left(M_{i}^{s}\left(\tilde{x}_{i}\right)\right)_{s \in \mathcal{S}}$. The resulting list of possible consumption plans is $\Pi\left[M_{i}\left(\tilde{x}_{i}\right)\right]$. If $x_{i} \in \Pi\left[M_{i}\left(\tilde{x}_{i}\right)\right]$, we either have a truthful delivery or a concealed violation of the contract.

In the model of Radner (1968), the consequence of incomplete information is a restriction of the choice set to $P_{i}$-measurable plans of consumption bundles. Here the consequences are less stringent. An agent can enforce delivery of $P_{i}$-measurable plans of lists (which include all $P_{i}$-measurable consumption plans), but this does not imply $P_{i}$-measurability of the resulting consumption plan.

\subsection{Prices of lists}

The prices of contingent goods are normalized to belong to the simplex:

\footnotetext{
${ }^{12}$ Here and everywhere below, $\mathbb{I F}(\cdot)$ denotes the set of finite and non-empty subsets.
} 


$$
p \in \Delta^{S L}=\left\{p \in \mathbb{R}_{+}^{S L}: \sum_{s \in \mathcal{S}} \sum_{l \in \mathcal{L}} p^{s l}=1\right\} .
$$

In economies with uncertain delivery, we must extend prices to the space of plans of lists:

$$
\tilde{p}:\left(\mathbb{F}\left(\mathbb{R}_{+}^{L}\right)\right)^{S} \longrightarrow \mathbb{R}_{+} .
$$

We do this by restricting equilibrium prices to satisfy a "fundamental value" assumption: the price of a plan of contingent lists must be equal to the price of the cheapest plan of consumption bundles that satisfies the requirements of the plan of lists. To present this assumption, it is convenient to define $\tilde{p}^{s}$ as the price of a list for delivery in state $s$.

\section{Assumption 1.}

Let the prices of contingent goods be $p \in \Delta^{S L}$. The price of a plan of contingent lists, $\tilde{x}_{i}$, is:

$$
\tilde{p}\left(\tilde{x}_{i}\right)=\sum_{s \in \mathcal{S}} \tilde{p}^{s}\left(\tilde{x}_{i}^{s}\right)=\sum_{s \in \mathcal{S}} \min _{x_{i}^{s} \in \tilde{x}_{i}^{s}}\left\{p^{s} \cdot x_{i}^{s}\right\}
$$

Under this assumption, the budget restriction can be written as:

$$
\tilde{B}_{i}(p)=\left\{\tilde{x}_{i} \in\left(\mathbb{F}\left(\mathbb{R}_{+}^{L}\right)\right)^{S}: \sum_{s \in \mathcal{S}} \min _{x_{i}^{s} \in \tilde{x}_{i}^{s}}\left\{p^{s} \cdot x_{i}^{s}\right\} \leq p \cdot e_{i}\right\} .
$$

\subsection{Deliveries and preferences}

When buying a plan of lists, agents form expectations about the resulting deliveries. These expectations, together with preferences over consumption plans, induce preferences over plans of lists. We start by making standard assumptions on preferences over consumption plans. Once we know the relationship between lists and deliveries, it will become clear how to obtain preferences over plans of lists from preferences over consumption plans.

\section{Assumption 2.}

Preferences over consumption plans are represented by an expected utility function, $U_{i}\left(x_{i}\right)=$ $\sum_{s \in \mathcal{S}} \mu^{s} u_{i}^{s}\left(x_{i}^{s}\right)$, where each state-dependent utility function, $u_{i}^{s}: \mathbb{R}_{+}^{L} \rightarrow \mathbb{R}$, is continuous, concave and strictly increasing. ${ }^{13}$

\footnotetext{
${ }^{13}$ By strictly increasing, we mean that $x^{s} \geq y^{s}$ and $x^{s} \neq y^{s}$ implies that $u_{i}^{s}\left(x^{s}\right)>u_{i}^{s}\left(y^{s}\right)$.
} 
We impose a precise relationship between the plans of lists that are chosen by the agents and the actual deliveries. We assume that the consumption plan that is delivered is one of the cheapest among those that the agent must accept. In case of a tie, agents receive the consumption plan with the highest utility among the cheapest. This is in the spirit of the mechanism design literature, where incentive compatibility conditions only need to be satisfied in equality (in case of indifference, the agent is assumed to select the action that is preferred by the principal).

The set of the cheapest consumption plans, at prices $p$, among those in $\Pi\left[M_{i}\left(\tilde{x}_{i}\right)\right]$, is denoted by $\tilde{Y}_{i}\left(\tilde{x}_{i}, p\right)$ :

$$
\begin{aligned}
& \tilde{Y}_{i}:\left(\mathbb{F}\left(\mathbb{R}_{+}^{L}\right)\right)^{S} \times \Delta^{S L} \longrightarrow \mathbb{F}\left(\mathbb{R}_{+}^{S L}\right) ; \\
& \tilde{Y}_{i}\left(\tilde{x}_{i}, p\right)=\left\{x_{i} \in \mathbb{R}_{+}^{S L}: x_{i} \in \underset{z_{i} \in \Pi\left[M_{i}\left(\tilde{x}_{i}\right)\right]}{\operatorname{argmin}}\left\{p \cdot z_{i}\right\}\right\} .
\end{aligned}
$$

\section{Assumption 3.}

For given prices of contingent goods, $p \in \Delta^{S L}$, if agent $i$ chooses the plan of lists $\tilde{x}_{i}$, the consumption plan that will be delivered is:

$$
x_{i} \in \underset{z_{i} \in \tilde{Y}_{i}\left(\tilde{x}_{i}, p\right)}{\operatorname{argmax}} U_{i}\left(z_{i}\right)
$$

Knowing this relationship, rational agents are able attribute to each plan of lists the utility of the consumption plan that will actually be delivered.

For agent $i$, the expected utility of a plan of lists, $\tilde{x}_{i}$, at prices $p$, is defined as:

$$
\begin{aligned}
& V_{i}:\left(\mathbb{F}\left(\mathbb{R}_{+}^{L}\right)\right)^{S} \times \Delta^{S L} \longrightarrow \mathbb{R} ; \\
& V_{i}\left(\tilde{x}_{i}, p\right)=\max _{x_{i} \in \tilde{Y}_{i}\left(\tilde{x}_{i}, p\right)} U_{i}\left(x_{i}\right) .
\end{aligned}
$$

The problem of agent $i$ can, therefore, be written as:

$$
\max _{\tilde{x}_{i} \in \tilde{B}_{i}(p)} V_{i}\left(\tilde{x}_{i}, p\right)
$$

Observe that adding to a list an alternative that is more expensive than those that are already in the list does not affect the price nor the utility of the list. Therefore, there are many plans of lists that solve that problem of the agent.

Suppose that agent $i$ chooses a plan of lists, $\tilde{x}_{i}$, with an anticipated delivery of $x_{i}$ (composed by the cheapest bundles in each state of nature). The agent would not mind changing his/her choice 
to $M_{i}\left(x_{i}\right)$ because the utility is the same as that of $\tilde{x}_{i}$ and the price is not higher. ${ }^{14}$ This change consists in removing the irrelevant alternatives in the lists (those that are never delivered, and, therefore, do not affect the price of the list).

\section{Equilibrium}

\subsection{Concept}

Recall that we are considering an economy with a finite number of agents, $\mathcal{I}=\{1, \ldots, I\}$, who trade (at date 0 ) a finite number of commodities, $\mathcal{L}=\{1, \ldots, L\}$, under uncertainty about which of a finite number of possible states of nature, $\mathcal{S}=\{1, \ldots, S\}$, will occur (at date 1 ).

At date 0, taking as given the prices of plans of lists, $\tilde{p}$, agent $i$ trades his/her contingent endowments, $e_{i} \in \mathbb{R}_{++}^{S L} \cap P_{i}$, for a plan of contingent lists, $\tilde{x}_{i}=\left(\tilde{x}_{i}^{1}, \tilde{x}_{i}^{2}, \ldots, \tilde{x}_{i}^{S}\right) \in\left(\mathbb{F}\left(\mathbb{R}_{+}^{L}\right)\right)^{S}$, that specifies the bundles that may be delivered in each state of nature. At date 1, if state $s$ occurs, agent $i$ receives a bundle $x_{i}^{s} \in M_{i}^{s}\left(\tilde{x}_{i}\right)=\bigcup_{t \in P_{i}(s)} \tilde{x}_{i}^{t}$.

When buying a plan of lists, $\tilde{x}_{i}$, agent $i$ expects to receive the cheapest consumption plan in $\Pi\left[M_{i}\left(\tilde{x}_{i}\right)\right]$. Therefore, the expected utility of the plan of lists is the expected utility of this cheapest consumption plan, $V_{i}\left(\tilde{x}_{i}, p\right)$.

In equilibrium, given prices $\tilde{p}^{*}$, each agent $i$ chooses an individually optimal plan of lists, $\tilde{x}_{i}^{*}$, and receives a consumption plan, $x_{i}^{*} \in \Pi\left[M_{i}\left(\tilde{x}_{i}^{*}\right)\right]$ that has the anticipated utility, $V_{i}\left(\tilde{x}_{i}^{*}, p^{*}\right)$, with the allocation, $\left\{x_{i}^{*}\right\}_{i \in \mathcal{I}}$, being feasible.

\section{Definition 1.}

An equilibrium of an economy with uncertain delivery, $\left(\tilde{x}^{*}, x^{*}, \tilde{p}^{*}\right)$, is composed by: plans of lists, $\tilde{x}^{*}=\left(\tilde{x}_{1}^{*}, \ldots, \tilde{x}_{I}^{*}\right) ;$ an allocation, $x^{*}=\left(x_{1}^{*}, \ldots, x_{I}^{*}\right)$; and a price system, $\tilde{p}^{*}$, whose restriction to consumption plans is $p^{*} \in \Delta^{S L}$. These are such that:

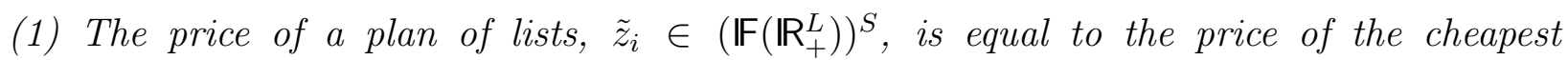
consumption plan that satisfies the requirements of the plan of lists, $\tilde{p}^{*}\left(\tilde{z}_{i}\right)=\sum_{s \in \mathcal{S}} \min _{z_{i}^{s} \in \tilde{z}_{i}^{s}}\left\{p^{s *} \cdot z_{i}^{s}\right\}$;

\footnotetext{
${ }^{14}$ Recall that $M_{i}\left(x_{i}\right)=\left(M_{i}^{s}\left(x_{i}\right)\right)_{s \in \mathcal{S}}$, with $M_{i}^{s}\left(x_{i}\right)=\bigcup_{t \in P_{i}(s)} x_{i}^{t}$.
} 
(2) In each state of nature, $s \in \mathcal{S}$, the bundle that is delivered to each agent $i \in \mathcal{I}$ is one of the cheapest that the agent has to accept, more precisely, $x_{i}^{*} \in \underset{z_{i} \in \tilde{Y}_{i}\left(\tilde{x}_{i}^{*}, p^{*}\right)}{\operatorname{argmax}} U_{i}\left(z_{i}\right)$;

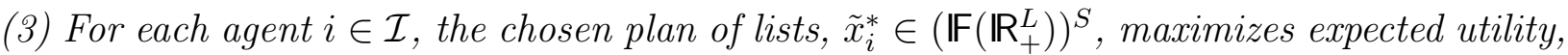
$V_{i}\left(\tilde{x}_{i}^{*}, p^{*}\right)$, in the agent's budget set, $\tilde{B}_{i}\left(p^{*}\right)=\left\{\tilde{z}_{i} \in\left(\mathbb{F}\left(\mathbb{R}_{+}^{L}\right)\right)^{S}: \tilde{p}^{*}\left(\tilde{z}_{i}\right) \leq p^{*} \cdot e_{i}\right\}$;

(4) The allocation, $x^{*} \in\left(\mathbb{R}_{+}^{S L}\right)^{I}$, is feasible, $\sum_{i \in \mathcal{I}} x_{i}^{*} \leq \sum_{i \in \mathcal{I}} e_{i}$.

Observe that the informational constraints are present: in condition (2), which establishes the (price-dependent) relationship between plans of lists that are bought and consumption plans that are delivered; and in condition (3), according to which each agent chooses a plan of lists by comparing the utility of the consumption plans that will be delivered. ${ }^{15}$

Notice also that condition (1) is simply a restatement of Assumption 1.

In the next section, we reformulate the problem of the agent from a choice over plans of lists to a choice over the consuption plans that result from the plans of lists that are chosen. This allows us to arrive at a formulation of the equilibrium concept that only refers to consumption plans (and not plans of lists) and that, for this reason, is more tractable.

\subsection{Deliverable consumption plans}

Suppose that agent $i$ buys a plan of singleton lists for delivery in two possible states of nature, $\tilde{x}_{i}=\left(x_{i}^{s}, x_{i}^{t}\right)$. If the agent can distinguish states $s$ and $t$, then $M_{i}\left(\tilde{x}_{i}\right)=\left(x_{i}^{s}, x_{i}^{t}\right)$, and thus delivery of $x_{i}^{s}$ in state $s$ and $x_{i}^{t}$ in state $t$ is guaranteed. But if the agent cannot distinguish the two states, then we have $M_{i}\left(\tilde{x}_{i}\right)=\left(\left\{x_{i}^{s}, x_{i}^{t}\right\},\left\{x_{i}^{s}, x_{i}^{t}\right\}\right)$. As a result: the agent receives, in state $s$, the cheapest of the two alternatives according to $p^{s}$; and, in state $t$, the cheapest according to $p^{t}$.

The bundle that is delivered in state $s$ cannot be more expensive (according to prices for delivery in state $s$ ) than any of the bundles promised for delivery in states $t \in P_{i}(s)$ :

$$
\forall t \in P_{i}(s), p^{s} \cdot x_{i}^{s} \leq p^{s} \cdot x_{i}^{t}
$$

\footnotetext{
${ }^{15}$ More formally, the information structure $P_{i}$ enters the definition of equilibrium in the following way. Recall that $V_{i}\left(\tilde{x}_{i}, p^{*}\right)=\max _{x_{i} \in \tilde{Y}_{i}\left(\tilde{x}_{i}, p^{*}\right)} U_{i}\left(x_{i}\right)$, where $\tilde{Y}_{i}\left(\tilde{x}_{i}, p^{*}\right)=\operatorname{argmin}_{z_{i} \in \Pi\left[M_{i}\left(\tilde{x}_{i}\right)\right]}\left\{p^{*} \cdot z_{i}\right\}$. In turn, $\Pi\left[M_{i}\left(\tilde{x}_{i}\right)\right]=$ $\Pi_{s \in \mathcal{S}} M_{i}^{s}\left(\tilde{x}_{i}\right)$, where $M_{i}^{s}\left(\tilde{x}_{i}\right)=\cup_{t \in P_{i}(s)} \tilde{x}_{i}^{t}$.
} 
The set of deliverable consumption plans depends on prices. This dependence is described by the deliverability correspondence, defined below.

\section{Definition 2.}

$$
\begin{aligned}
& C_{i}: \Delta^{S L} \rightarrow \mathbb{R}_{+}^{S L} ; \\
& C_{i}(p)=\left\{x_{i} \in \mathbb{R}_{+}^{S L}: \forall s \in \mathcal{S}, p^{s} \cdot x_{i}^{s}=\min _{t \in P_{i}(s)}\left\{p^{s} \cdot x_{i}^{t}\right\}\right\} .
\end{aligned}
$$

A consumption plan is deliverable, $x_{i} \in C_{i}(p)$, if and only if there exists a $P_{i}$-measurable plan of lists of which $x_{i}$ is the cheapest alternative. It is enough to check whether or not $x_{i}$ is the cheapest alternative in the list $M_{i}\left(x_{i}\right)$.

We can formulate the problem of the agent, equivalently, as a choice over lists in $\tilde{B}_{i}(p)$ or as a choice over consumption plans in $B_{i}(p) \cap C_{i}(p)$, with $B_{i}(p)=\left\{x_{i} \in \mathbb{R}_{+}^{S L}: p \cdot x_{i} \leq p \cdot e_{i}\right\}$. The following results make this precise.

\section{Claim 1.}

Let $\tilde{p}$ be a price system that satisfies Assumption 1. Then:

$$
\tilde{x}_{i} \in \underset{\tilde{z}_{i} \in \tilde{B}_{i}(\tilde{p})}{\operatorname{argmax}} V_{i}\left(\tilde{z}_{i}, p\right) \Rightarrow \exists x_{i} \in \Pi\left[M_{i}\left(\tilde{x}_{i}\right)\right] \text { s.t. } U_{i}\left(x_{i}\right)=V_{i}\left(\tilde{x}_{i}, p\right) \wedge x_{i} \in \underset{x_{i} \in B_{i}(p) \cap C_{i}(p)}{\operatorname{argmax}} U_{i}\left(x_{i}\right) .
$$

\section{Claim 2.}

Let $\tilde{p}$ be a price system that satisfies Assumption 1. Then:

$$
x_{i} \in \underset{x_{i} \in B_{i}(p) \cap C_{i}(p)}{\operatorname{argmax}} U_{i}\left(x_{i}\right) \Rightarrow M_{i}\left(x_{i}\right) \in \underset{\tilde{x}_{i} \in \tilde{B}_{i}(\tilde{p})}{\operatorname{argmax}} V_{i}\left(\tilde{x}_{i}, p\right) .
$$

This equivalence allows us to reformulate the notion of equilibrium.

\section{Definition 3.}

A fundamental equilibrium of an economy with uncertain delivery, $\left(x^{*}, p^{*}\right)$, is composed by an allocation, $x^{*}=\left(x_{1}^{*}, \ldots, x_{I}^{*}\right)$ and a price system, $p^{*} \in \Delta^{S L}$, which are such that: 
(1) Each agent's choice is optimal, $x_{i}^{*} \in \underset{x_{i} \in B_{i}\left(p^{*}\right) \cap C_{i}\left(p^{*}\right)}{\operatorname{argmax}} U_{i}\left(x_{i}\right)$;

(2) The allocation, $x^{*}$, is feasible. That is, $\sum_{i \in \mathcal{I}} x_{i}^{*} \leq \sum_{i \in \mathcal{I}} e_{i}$.

In this reformulation, we removed the reference to lists because their prices can be obtained (by Assumption 1) from the prices of contingent goods and because optimal plans of lists for the agents to choose are simply given by $M_{i}\left(x_{i}^{*}\right), \forall i \in \mathcal{I}$.

\section{Claim 3.}

If $\left(\tilde{x}^{*}, x^{*}, p^{*}\right)$ is an equilibrium of an economy with uncertain delivery, then $\left(x^{*}, p^{*}\right)$ is a fundamental equilibrium.

\section{Claim 4.}

If $\left(x^{*}, p^{*}\right)$ is a fundamental equilibrium of an economy with uncertain delivery, then $\left(M\left(x^{*}\right), x^{*}, p^{*}\right)$, with $M\left(x^{*}\right)=\left(M_{i}\left(x_{i}^{*}\right)\right)_{i \in \mathcal{I}}$, is an equilibrium.

This alternative definition of equilibrium does not use preferences over lists, $V_{i}$, nor prices over lists, $\tilde{p}$. Therefore, we can compare it with the equilibrium with public state verification (ArrowDebreu-McKenzie under uncertainty) and the equilibrium with differential information proposed by Radner (1968). Everything boils down to the choice sets. In Arrow-Debreu-McKenzie: $X_{i}^{A D}=\mathbb{R}_{+}^{S L}$; in Radner (1968): $X_{i}^{R}=\mathbb{R}_{+}^{S L} \cap P_{i}$; in an economy with uncertain delivery: $X_{i}(p)=\mathbb{R}_{+}^{S L} \cap C_{i}(p)$. It should be clear that, for all $p \in \Delta^{S L}, X_{i}^{R} \subseteq X_{i}(p) \subseteq X_{i}^{A D}$.

\section{Existence of equilibrium}

If the correspondence from prices to the deliverable budget set, $B_{i}(p) \cap C_{i}(p)$, were continuous, establishing existence of equilibrium would be straightforward. But, as we illustrate in Appendix $3, C_{i}(p)$ is not lower hemicontinuous (this property fails when prices in some state are null, or when prices in two undistinguished states are collinear). 


\subsection{A sequence of economies}

In order to establish existence of equilibrium, we construct a sequence of economies. In these economies, the choice set is not constrained to satisfy the deliverability conditions. But violating these constraints implies an utility penalty. The penalty is a function of the difference between the cheapest consumption plan and the consumption plan that is delivered. ${ }^{16}$

In the economy $\mathcal{E}^{j}$, if state $s$ occurs, the utility penalty imposed on agent $i$ is:

$$
Z_{i}^{s j}\left(x_{i}, p\right)=j \max _{t \in P_{i}(s)}\left\{p^{s} \cdot x_{i}^{s}-p^{s} \cdot x_{i}^{t}\right\}
$$

Since $s \in P_{i}(s)$, the maximum is at least zero, thus penalties are never negative. Penalties are scaled up along the sequence of economies, and this is actually the only difference among the economies in the sequence.

In the economy $\mathcal{E}^{j}$, the utility functions of the agents are:

$$
U_{i}^{j}\left(x_{i}, p\right)=U_{i}\left(x_{i}\right)-j \sum_{s \in \mathcal{S}} \mu^{s} \max _{t \in P_{i}(s)}\left\{p^{s} \cdot x_{i}^{s}-p^{s} \cdot x_{i}^{t}\right\}
$$

For any $j \in \mathbb{N}$, the utility functions are continuous in consumption plans and prices, $\left(x_{i}, p\right) \in$ $\mathbb{R}_{+}^{S L} \times \Delta^{S L}$. The maximum of linear functions is a convex function, and multiplying a convex function by a negative constant, $-j$, yields a concave function. Hence, the objective function, $U_{i}^{j}\left(x_{i}, p\right)$, is concave in the first variable. Observe also that the utility penalty preserves the property of no satiation. The plan $x_{i}+\epsilon \overline{1}$ is always preferred to $x_{i}$ (the utility penalty is kept constant). The fact that the utility functions depend (continuously) on prices does not interfere with existence of equilibrium. ${ }^{17}$

\section{Lemma 1.}

Let $\mathcal{E}^{j}$ be an Arrow-Debreu-McKenzie economy in which each agent $i \in \mathcal{I}$ :

- has strictly positive initial endowments, $e_{i} \in \mathbb{R}_{++}^{S L}$;

- maximizes the utility function $U_{i}^{j}\left(x_{i}, p\right)=U_{i}\left(x_{i}\right)-j \sum_{s \in \mathcal{S}} \mu^{s} \max _{t \in P_{i}(s)}\left\{p^{s} \cdot x_{i}^{s}-p^{s} \cdot x_{i}^{t}\right\}$, with $U_{i}\left(x_{i}\right)$ continuous, concave and strictly increasing.

\footnotetext{
${ }^{16}$ These economies are an artifice to establish existence of equilibrium.

${ }^{17}$ With price dependent preferences, it is known that equilibrium exists (Arrow and Hahn, 1971). In the context of economies with uncertain delivery, see Correia-da-Silva and Hervés-Beloso (2008).
} 
Then, there exists an Arrow-Debreu-McKenzie equilibrium, $\left(x^{j}, p^{j}\right)$.

The sequence of economies, $\left\{\mathcal{E}^{j}\right\}_{j \in \mathbf{N}}$, has a sequence of equilibria, $\left\{\left(x^{j}, p^{j}\right)\right\}_{j \in \mathbf{N}}$, that lies in the compact set $\left[0, e_{T}\right]^{I} \times \Delta^{S L}$, where $e_{T}=\sum_{i \in \mathcal{I}} e_{i}$. There exists a subsequence that converges. In order to show that the limit, $\left(x^{*}, p^{*}\right)$, is a fundamental equilibrium of the original economy with uncertain delivery, we must prove that the following conditions are satisfied:

(1) Feasibility: $\sum_{i \in \mathcal{I}} x_{i}^{*} \leq \sum_{i \in \mathcal{I}} e_{i}$;

(2) Budget restriction: $\forall i \in \mathcal{I}, p^{*} \cdot x_{i}^{*} \leq p^{*} \cdot e_{i}$;

(3) Deliverability: $\forall i \in \mathcal{I}, x_{i}^{*} \in C_{i}\left(p^{*}\right)$;

(4) Individual optimality: $\forall i \in \mathcal{I}, x_{i} \in B_{i}\left(p^{*}\right) \cap C_{i}\left(p^{*}\right) \Rightarrow U_{i}\left(x_{i}^{*}\right) \geq U_{i}\left(x_{i}\right)$.

It is easy to show that the first three conditions are satisfied.

\section{Lemma 2.}

Consider a sequence of economies $\left\{\mathcal{E}^{j}\right\}_{j \in \mathbb{N}}$ defined as in Lemma 1, and a corresponding sequence of equilibria, $\left\{\left(x^{j}, p^{j}\right)\right\}_{j \in \mathbb{N}}$.

Then, the sequence of equilibria has an accumulation point, $\left(x^{*}, p^{*}\right)$, that satisfies:

(1) Feasibility: $\sum_{i \in \mathcal{I}} x_{i}^{*} \leq \sum_{i \in \mathcal{I}} e_{i}=e_{T}$;

(2) Budget restriction: $\forall i \in \mathcal{I}, p^{*} \cdot x_{i}^{*} \leq p^{*} \cdot e_{i}$;

(3) Deliverability: $\forall i \in \mathcal{I}, x_{i}^{*} \in C_{i}\left(p^{*}\right)$.

The difficult part of the proof is to verify condition (4): that the limit, $\left(x^{*}, p^{*}\right)$, maximizes utility in the deliverable budget set, $B_{i}\left(p^{*}\right) \cap C_{i}\left(p^{*}\right)$. The fact that $C_{i}$ is not lower hemicontinuous (as shown in Appendix 3) could prevent $\left(x^{*}, p^{*}\right)$ from being optimal. There could be a deliverable consumption plan $y_{i} \in B_{i}\left(p^{*}\right) \cap C_{i}\left(p^{*}\right)$ that is not even nearly deliverable in the economies in the sequence. In spite of having a low utility level for high $j$ (because of the penalty), this plan could be optimal in the original economy, and, in this case, $\left(x^{*}, p^{*}\right)$ would not be an equilibrium (an example of non-existence of equilibrium is given in Appendix 2).

We start by showing that, if any state of nature, $s \in \mathcal{S}$ can be verified by at least one of the agents, then $p^{*}$ is strictly positive. 
Lemma 3. Consider a sequence of economies $\left\{\mathcal{E}^{j}\right\}_{j \in \mathbb{N}}$ defined as in Lemma 1, a corresponding sequence of equilibria, $\left\{\left(x^{j}, p^{j}\right)\right\}_{j \in \mathbb{N}}$, and an accumulation point, $\left(x^{*}, p^{*}\right)$.

Suppose that for every $s \in \mathcal{S}$, there exists $i \in \mathcal{I}$ with $P_{i}(s)=\{s\}$. Then, $p^{*} \gg 0$.

\subsection{The existence result}

If agents have $P_{i}$-measurable endowments and preferences, and if any state can be verified by at least one of the agents, existence of equilibrium is guaranteed.

\section{Theorem 1.}

Consider an economy with uncertain delivery, $\mathcal{E} \equiv\left(e_{i}, u_{i}, \mu_{i}, P_{i}\right)_{i \in \mathcal{I}}$, such that:

- Preferences are represented by an expected utility function, $U_{i}\left(x_{i}\right)=\sum_{s \in \mathcal{S}} \mu^{s} u_{i}^{s}\left(x_{i}^{s}\right)$, where each $u_{i}^{s}: \mathbb{R}_{+}^{L} \rightarrow \mathbb{R}$ is continuous, concave and strictly increasing;

- Contingent preferences are the same in indistinguished states, $t \in P_{i}(s) \Rightarrow u_{i}^{t}(\cdot)=u_{i}^{s}(\cdot)$;

- Initial endowments are strictly positive and constant across indistinguished states: $e_{i} \in$ $\mathbb{R}_{++}^{S L} \cap P_{i}=\left\{e_{i} \in \mathbb{R}_{++}^{S L}: t \in P_{i}(s) \Rightarrow e_{i}^{t}=e_{i}^{s}\right\}$

- For each $s \in \mathcal{S}$, there exists $i \in \mathcal{I}$ with $P_{i}(s)=\{s\}$.

Then, there exists an equilibrium of the economy with uncertain delivery.

The strategy of the proof is to assume (by way of contradiction) that there exists a $x_{i}^{\prime}$ in $B_{i}\left(p^{*}\right) \cap C_{i}\left(p^{*}\right)$ that is preferred to $x_{i}^{*}$, and then find that there exists a similar $x_{i}$ which belongs to $B_{i}\left(p^{j}\right) \cap C_{i}\left(p^{j}\right)$, for large $j$. This contradicts that $\left(x^{j}, p^{j}\right)$ is an equilibrium of $\mathcal{E}^{j}$, because $x_{i}$ would also be preferred to $x_{i}^{j}$ in the economy $\mathcal{E}^{j}$.

\subsection{Incentive compatibility}

One may wonder whether agents may have an incentive to misrepresent their information. In some state $s \in \mathcal{S}$, a coalition of agents $G \subset \mathcal{I}$ may wish to send false reports which imply that the net trades that were agreed for state $t$ are carried out instead of those that were agreed for 
state $s$. This will be the case if it all the agents gain with the deviation, $u_{i}^{s}\left(e_{i}^{s}+x_{i}^{t}-e_{i}^{t}\right)>$ $u_{i}^{s}\left(x_{i}^{s}\right), \forall i \in G$, if the false reports do not contradict the supposedly truthful reports of the other agents, $t \in \cap_{j \in \mathcal{I} \backslash G} P_{j}(s)$, and if it is common knowledge among the members of the coalition that they should send false reports, $P_{i}(s)=P_{j}(s), \forall(i, j) \in G^{2}$. If this is never the case, it is said that the allocation satisfies weak coalitional incentive compatibility (Koutsougeras and Yannelis, 1993).

\section{Definition 4 .}

An allocation, $x=\left(x_{1}, \ldots, x_{I}\right)$, satisfies weak coalitional incentive compatibility if and only if it is not true that in some state, $s \in \mathcal{S}$, there exists a coalition, $G \subset \mathcal{I}$, whose members agree that they should deviate, $P_{i}(s)=P_{j}(s), \forall(i, j) \in G^{2}$, and an undetectable deviation, $t \in \cap_{k \in \mathcal{I} \backslash G} P_{k}(s)$, with which all the members of the coalition become better off, $u_{i}^{s}\left(e_{i}^{s}+x_{i}^{t}-e_{i}^{t}\right)>u_{i}^{s}\left(x_{i}^{s}\right), \forall i \in G$.

In the conditions of Theorem 1, we find that an equilibrium allocation always satisfies weak coalitional incentive compatibility.

\section{Theorem 2.}

Consider an economy with uncertain delivery, $\mathcal{E} \equiv\left(e_{i}, u_{i}, \mu_{i}, P_{i}\right)_{i \in \mathcal{I}}$, that satisfies the conditions of Theorem 1.

If $x^{*}$ is an equilibrium allocation, then it satisfies weak coalitional incentive compatibility.

To understand why weak coalitional incentive compatibility holds ${ }^{18}$, suppose that, in state $s$, the agents in a coalition send false reports (that do not contradict the truthful reports of the other agents) which imply that the net trades that are carried out are those that were agreed for state $t$. The fact that the agents outside the coalition cannot distinguish state $s$ from $t$ implies that they must have the same endowments in both states and must receive a net trade in state $t$ that is at least as expensive (according to prices $p^{s *}$ ) as the net trade that was contracted for state $s$ (because of the deliverability constraints). Since the coalition receives an aggregate net trade that is symmetric to the aggregate net trade of the agents outside the coalition, we conclude that the coalition ends up receiving a net trade that is not more expensive (again, according to prices $\left.p^{s *}\right)$ than the contracted one. But a cheaper net trade could never yield more utility to an agent of the coalition, as this would contradict the fact that the initial choice maximized utility (for

\footnotetext{
${ }^{18} \mathrm{~A}$ formal proof is presented in Appendix 1.
} 
all agents in the coalition, utility maximization for state $s$ is not subject to any deliverability constraint because $\left.P_{i}(s)=\{s\}\right)$.

A stronger incentive compatibility property dispenses the requirement that the agents agree that they should deviate, $P_{i}(s)=P_{j}(s), \forall(i, j) \in G^{2}$. It is not guaranteed that an equilibrium allocation satisfies this stronger notion, designated as coalitional incentive compatibility by Koutsougeras and Yannelis (1993). ${ }^{19}$

\section{Concluding remarks}

In our model of trade ex ante with differential information, agents make contingent trade contracts before receiving their private information, and then use their private information to enforce contracts. A contract attributes a list of possible consumption bundles to each element of the agent's information partition.

Agents must, then, form expectations about which of the bundles in the list they will actually consume in each of the states of nature. In previous works (2008, 2009), we have considered two distinct cases: (i) agents expected to receive the bundle with the lowest possible utility (prudent expectations); and (ii) agents formed a probability belief about the delivery that was some continuous function of both the elements of the list and of prices (subjective expectations).

However, in our model agents always receive, in each state of nature, the cheapest consumption bundle among those in a list. Therefore, it is more consistent (and it is what we do in this paper) to assume that agents expect to receive, in each state of nature, the cheapest consumption bundle among those in a list (rational expectations).

To study this more interesting case, we showed that our setup in which agents choose among plans of lists can be translated into the more typical setup in which agents choose among consumption plans. All that is necessary is to modify the choice correspondence of the agents, restricting it to what we called the deliverability correspondence. This choice set always includes the set of $P_{i}$-measurable consumption plans.

The fact that the deliverability correspondence is not lower hemicontinuous prevents us from

\footnotetext{
${ }^{19}$ Krasa and Yannelis (1994) designate it as weak coalitional incentive compatibility to distinguish it from a stronger notion in which endowment redistributions among the members of the coalition are permitted.
} 
applying Berge's Maximum theorem to establish existence of equilibrium. In fact, with agents having rational expectations, existence of equilibrium is no longer guaranteed. To establish existence of equilibrium we needed to assume that any state of nature can be perfectly verified by at least one agent. Most of the technical work in this paper is devoted to the existence proof, which is based on the construction of a sequence of Arrow-Debreu economies whose limit is similar to our differential information economy.

A central issue in most models of trade with differential information is whether agents have the incentives to reveal their private information in a truthful way. We find that, given an equilibrium allocation, if agents could misrepresent their information, the assumption that any state of nature can be perfectly verified by at least one agent guarantees that no coalition is able to gain by sending false reports.

Our final remarks address two crucial assumptions that are made in this paper. One is that the information provided by the agents to enforce contracts is not aggregated by any institution. Otherwise, such an institution could announce the true state of nature, and state verification would become public. Another assumption is that agents cannot trade after receiving their information. The study of the consequences of removing this restriction is left for future research. 


\section{Appendix 1: The proofs}

\section{Proof of Claim 1:}

Let $x_{i}=\underset{z_{i} \in \tilde{Y}_{i}\left(\tilde{x}_{i}, p\right)}{\operatorname{argmax}} U_{i}\left(z_{i}\right)$. Such $x_{i}$ is an element of $\Pi\left[M_{i}\left(\tilde{x}_{i}\right)\right]$ and is such that $U_{i}\left(x_{i}\right)=V_{i}\left(\tilde{x}_{i}, p\right)$.

Suppose that there exists $y_{i} \in B_{i}(p) \cap C_{i}(p)$ that is preferred to $x_{i}: U_{i}\left(y_{i}\right)>U_{i}\left(x_{i}\right)$.

Since $y_{i} \in C_{i}(p): V_{i}\left(M_{i}\left(y_{i}\right), p\right) \geq U_{i}\left(y_{i}\right)>U_{i}\left(x_{i}\right)=V_{i}\left(\tilde{x}_{i}, p\right)$.

From Assumption 1: $\tilde{p}\left(M_{i}\left(y_{i}\right)\right) \leq p \cdot y_{i}$. Therefore, $M_{i}\left(y_{i}\right) \in \tilde{B}_{i}(\tilde{p})$. Contradiction.

\section{Proof of Claim 2:}

We know that $V_{i}\left(M_{i}\left(x_{i}\right), p\right)=U_{i}\left(x_{i}\right)$.

Suppose that there exists $\tilde{y}_{i} \in \tilde{B}_{i}(p)$ that is preferred to $M_{i}\left(x_{i}\right): V_{i}\left(\tilde{y}_{i}, p\right)>V_{i}\left(M_{i}\left(x_{i}\right), p\right)$.

Let $y_{i}=\underset{z_{i} \in \tilde{Y}_{i}\left(\tilde{y}_{i}, p\right)}{\operatorname{argmax}} U_{i}\left(z_{i}\right)$. Then: $U_{i}\left(y_{i}\right)=V_{i}\left(\tilde{y}_{i}, p\right)>V_{i}\left(M_{i}\left(x_{i}\right), p\right)=U_{i}\left(x_{i}\right)$.

From Assumption 1: $\tilde{y}_{i} \in \tilde{B}_{i}(p) \Rightarrow y_{i} \in B_{i}(p)$. Contradiction.

\section{Proof of Claim 3:}

The proof follows directly from Definitions 1 and 3 and from Claim 1.

\section{Proof of Claim 4:}

The proof follows directly from Definitions 1 and 3 and from Claim 2.

\section{Proof of Lemma 1:}

Restrict the choice set to the compact $[0, T]$, with $T=2 \sum_{i \in \mathcal{I}} e_{i}$.

Consider correspondences, $\left\{\psi_{i}\right\}_{i \in \mathcal{I}}$, which assign to given prices, $p$, consumption plans, $x_{i}^{\prime}$, that maximize $U_{i}^{j}\left(x_{i}, p\right)$ in the restricted budget set, $\bar{B}_{i}(p)=B_{i}(p) \cap[0, T]$ :

$$
\begin{aligned}
& \psi_{i}:[0, T]^{I} \times \Delta^{S L} \longrightarrow[0, T] ; \\
& x_{i}^{\prime} \in \psi_{i}(x, p) \Leftrightarrow x_{i}^{\prime} \in \underset{x_{i} \in \bar{B}_{i}(p)}{\operatorname{argmax}} U_{i}^{j}\left(x_{i}, p\right) .
\end{aligned}
$$

Consider also a correspondence, $\psi_{p}$, that assigns to the total demand, $\sum_{i \in \mathcal{I}} x_{i}$, the prices, $p^{\prime}$, which maximize the value of excess demand: 


$$
\begin{aligned}
& \psi_{p}:[0, T]^{I} \times \Delta^{S L} \longrightarrow \Delta^{S L} \\
& p^{\prime} \in \psi_{p}(x, p) \Leftrightarrow p^{\prime} \in \underset{p \in \Delta^{S L}}{\operatorname{argmax}}\left\{p \cdot \sum_{i \in \mathcal{I}}\left(x_{i}-e_{i}\right)\right\} .
\end{aligned}
$$

The objective functions, $\left\{U_{i}^{j}\right\}_{i \in \mathcal{I}}$ and $p \cdot \sum_{i \in \mathcal{I}}\left(x_{i}-e_{i}\right)$, are continuous, and $\bar{B}_{i}(p)$ is a continuous correspondence. We can, therefore, use Berge's Maximum Theorem to show that each of the correspondences, $\left\{\psi_{i}\right\}_{i \in \mathcal{I}}$ and $\psi_{p}$, is upper hemicontinuous with non-empty and compact values. They also have convex values because the objective functions are concave. The product correspondence retains these properties and maps a compact set into itself:

$$
\begin{aligned}
& \psi \equiv \prod_{i=1}^{I} \psi_{i} \times \psi_{p} \\
& \psi:[0, T]^{I} \times \Delta^{S L} \longrightarrow[0, T]^{I} \times \Delta^{S L} ; \\
& \left(x^{\prime}, p^{\prime}\right) \in \psi(x, p) \Leftrightarrow x_{i}^{\prime} \in \psi_{i}(x, p), \forall i \in \mathcal{I} \text { and } p^{\prime} \in \psi_{p}(x, p) .
\end{aligned}
$$

Existence of a fixed-point, $\left(x^{*}, p^{*}\right)$, follows from Kakutani's Theorem.

It is clear that $x_{i}^{*}$ solves the problem of agent $i$.

The fact that $p^{*}$ maximizes the value of excess demand implies that:

$$
p^{\prime} \cdot \sum_{i \in \mathcal{I}}\left(x_{i}^{*}-e_{i}\right) \leq p^{*} \cdot \sum_{i}\left(x_{i}^{*}-e_{i}\right) \leq 0, \text { for all } p^{\prime} \in \Delta^{S L} .
$$

Making $p^{\prime}=e^{s l}=(0, \ldots, 1, \ldots, 0)$, for each $(s, l) \in \mathcal{S} \times \mathcal{L}$, shows that $x^{*}$ is feasible: $\sum_{i \in \mathcal{I}}\left(x_{i}^{*}-e_{i}\right) \leq 0$.

The usual extension from $[0, T]^{I}$ to $\left(\mathbb{R}_{+}^{S L}\right)^{I}$ applies.

\section{Proof of Lemma 2:}

Consider only the subsequence that converges to $\left(x^{*}, p^{*}\right)$. Ignore the remaining terms of the sequence.

(1) The set of feasible allocations is closed, and the limit allocation, $\left\{x_{i}^{*}\right\}_{i \in \mathcal{I}}$, is the limit of a sequence of feasible allocations, therefore it is feasible $\left(\sum_{i \in \mathcal{I}} x_{i}^{*} \leq \sum_{i \in \mathcal{I}} e_{i}\right)$.

(2) the limit allocation is the limit of a sequence of allocations in the sequence of budget sets $\left(x_{i}^{j} \in\right.$ $\left.B_{i}\left(p^{j}\right), \forall i \in \mathcal{I}\right)$, therefore, it also belongs to the limit budget set $\left(x_{i}^{*} \in B_{i}\left(p^{*}\right), \forall i \in \mathcal{I}\right)$.

Suppose that $x_{i}^{*}$ does not satisfy the budget restriction of agent $i$. Let $\alpha=2\left\|e_{T}\right\|+1$, and select $\epsilon>0$ such that $p^{*} \cdot x_{i}^{*}-p^{*} \cdot e_{i}=\alpha \epsilon$. Choosing a sufficiently high $j$, we can guarantee that $\left\|x^{*}-x^{j}\right\|<\epsilon$ and $\left\|p^{*}-p^{j}\right\|<\epsilon$. With $p^{j}=p^{*}+d p, x^{j}=x_{i}^{*}+d x_{i}$, and manipulating:

$$
\left(p^{*}+d p\right) \cdot\left(x_{i}^{*}+d x_{i}\right)-\left(p^{*}+d p\right) \cdot e_{i}=p^{*} \cdot x_{i}^{*}-p^{*} \cdot e_{i}+p^{*} \cdot d x_{i}+d p \cdot x_{i}^{*}+d p \cdot d x_{i}-d p \cdot e_{i}=
$$




$$
=\alpha \epsilon+\left(p^{*}+d p\right) \cdot d x_{i}+d p \cdot\left(x_{i}^{*}-e_{i}\right)>\alpha \epsilon-\epsilon-\epsilon \cdot 2\left\|e_{T}\right\|=0 .
$$

This means that $x_{i}^{j} \notin B_{i}\left(p^{j}\right)$. Contradiction.

(3) To verify that the limit allocation, $x^{*}$, satisfies the deliverability restrictions in the limit economy, suppose that $x_{i}^{*}$ violated one of the restrictions by more than $\delta>0$. then, for sufficiently high $j, x_{i}^{j}$ would also violate the same restriction by more than $\delta$. For $t \in P_{i}(s), \exists j_{0} \in \mathbb{N}$ :

$$
p^{s *} \cdot x_{i}^{s *}>p^{s *} \cdot x_{i}^{t *}+\delta \Rightarrow p^{s j} \cdot x_{i}^{s j}>p^{s j} \cdot x_{i}^{t j}+\delta, \text { for all } j>j_{0} .
$$

Utility among feasible allocations is bounded by $U_{i}\left(e_{T}\right)$, so we can consider a $j$ that is sufficiently high for $j \delta>U_{i}\left(e_{T}\right)-U_{i}\left(e_{i}\right)$. It would follow that $U_{i}^{j}\left(x_{i}^{j}\right)<U_{i}\left(x_{i}^{j}\right)-j \delta<U_{i}\left(x_{i}^{j}\right)-U_{i}\left(e_{T}\right)+U_{i}\left(e_{i}\right)<$ $U_{i}\left(e_{i}\right)=U_{i}^{j}\left(e_{i}\right)$. Contradiction.

\section{Proof of Lemma 3:}

Consider a subsequence of equilibria, $\left\{\left(x^{n}, p^{n}\right)\right\}_{n \in \mathbb{N}}$, that converges to $\left(x^{*}, p^{*}\right)$, and assume (by way of contradiction) that $\exists(s, l) \in \mathcal{S} \times \mathcal{L}: p^{s l *}=0$.

Consider agent $i$ with $P_{i}(s)=\{s\}$ and his/her optimal choices, $x_{i}^{n} \in \underset{z \in B_{i}\left(p^{n}\right)}{\operatorname{argmax}} U_{i}^{n}(z)$.

We know that $x_{i}^{n} \leq e_{T}, \forall n \in \mathbb{N}$. Then, $x_{i}^{*} \leq e_{T}$.

Observe that $u_{i}^{s}\left(z^{s}\right)>u_{i}^{s}\left(x_{i}^{s}\right) \Rightarrow p^{s} n \cdot z^{s}>p^{s} n \cdot x_{i}^{s}$, otherwise $z^{s}$ would have been chosen instead of $x_{i}^{s}{ }^{n}$ in the plan $x_{i}^{n}$ (this is only true because $P_{i}(s)=\{s\}$ ).

Define $y_{i}^{s}$ by adding some of the free good, $(s, l)$, to $x_{i}^{s}$. By strict monotonicity: $u_{i}^{s}\left(y_{i}^{s}\right)>u_{i}^{s}\left(x_{i}^{s}\right)$. By continuity, there exists some $\alpha \in(0,1)$ such that $u_{i}^{s}\left(\alpha y_{i}^{s}\right)>u_{i}^{s}\left(x_{i}^{s}\right)$. For large $n$, we also have $u_{i}^{s}\left(\alpha y_{i}^{s}\right)>u_{i}^{s}\left(x_{i}^{s}{ }^{n}\right)$. This implies that $p^{s n} \cdot\left(\alpha y_{i}^{s}\right)>p^{s n} \cdot x_{i}^{s}{ }^{n}$, and, in the limit: $p^{s} \cdot\left(\alpha y_{i}^{s}\right) \geq p^{s} \cdot x_{i}^{s}$. Therefore, $p^{s} \cdot y_{i}^{s}>p^{s} \cdot x_{i}^{s}$. Contradiction.

\section{Proof of Theorem 1:}

Given Lemmas 1 and 2, all that is left to prove is (4), which states that the limit of the sequence of equilibria, $\left(x^{*}, p^{*}\right)$, is composed by optimal choices in the original economy with uncertain delivery, that is: $x_{i} \in B_{i}\left(p^{*}\right) \cap C_{i}\left(p^{*}\right) \Rightarrow U_{i}\left(x_{i}^{*}\right) \geq U_{i}\left(x_{i}\right), \forall i \in \mathcal{I}$.

By Lemma 3, we are sure that $p^{*} \gg 0$.

Assume (by way of contradiction) that there exists $y_{i} \in B_{i}\left(p^{*}\right) \cap C_{i}\left(p^{*}\right)$ such that $U_{i}\left(y_{i}\right)>U_{i}\left(x_{i}^{*}\right)$. We will show that this implies that $\left(x^{j}, p^{j}\right)$ is not an equilibrium of $\mathcal{E}^{j}$, for high $j$.

$\underline{\text { A preliminary remark }}$ 
Suppose that prices for delivery in $s$ and in $t \in P_{i}(s)$ are parallel: $p^{* s}=a p^{* t}$. The two deliverability conditions that involve prices $p^{* s}$ and $p^{* t}$ yield equalities:

$$
\left\{\begin{array} { l } 
{ p ^ { * s } \cdot y _ { i } ^ { s } \leq p ^ { * s } \cdot y _ { i } ^ { t } } \\
{ p ^ { * t } \cdot y _ { i } ^ { t } \leq p ^ { * t } \cdot y _ { i } ^ { s } }
\end{array} \Leftrightarrow \left\{\begin{array} { l } 
{ a p ^ { * t } \cdot y _ { i } ^ { s } \leq a p ^ { * t } \cdot y _ { i } ^ { t } } \\
{ p ^ { * t } \cdot y _ { i } ^ { t } \leq p ^ { * t } \cdot y _ { i } ^ { s } }
\end{array} \Leftrightarrow \left\{\begin{array}{l}
p^{* s} \cdot y_{i}^{s}=p^{* s} \cdot y_{i}^{t} \\
p^{* t} \cdot y_{i}^{t}=p^{* t} \cdot y_{i}^{s} .
\end{array}\right.\right.\right.
$$

The two consumption bundles, $y_{i}^{s}$ and $y_{i}^{t}$, cost the same in both states. Since $t \in P_{i}(s)$, we have $u_{i}^{s}=u_{i}^{t}$. If $u_{i}^{s}\left(y_{i}^{s}\right)>u_{i}^{s}\left(y_{i}^{t}\right)$, then the agent would be better off selecting $y_{i}^{s}$ for consumption in both states. Thus, we must have $u_{i}^{s}\left(y_{i}^{s}\right)=u_{i}^{s}\left(y_{i}^{t}\right)$. Since the utility functions are concave, the agent is not worse off consuming the average bundle in both states. Notice that if the original vector satisfies the deliverability conditions, then this average vector also does.

Construct $x_{i}^{\prime \prime}$ by modifying $y_{i}$, considering the average bundle whenever there are parallel prices. Therefore, we have $x_{i}^{\prime \prime s}=x_{i}^{\prime \prime t}$ whenever $p^{* s}=a p^{* t}$.

\section{Strategy of the proof}

Reformulating, we assume (by way of contradiction) that there exists a $x_{i}^{\prime \prime} \in B_{i}\left(p^{*}\right) \cap C_{i}\left(p^{*}\right)$ such that $U_{i}\left(x_{i}^{\prime \prime}\right)>U_{i}\left(x_{i}^{*}\right)$, with $x_{i}^{\prime \prime s}=x_{i}^{\prime \prime t}$ whenever $p^{* s}=a p^{* t}$.

By continuity of $U_{i}$, there exists $\delta>0$ such that $x_{i}^{\prime}=(1-\delta) x_{i}^{\prime \prime}$ is strictly preferred to $x_{i}^{*}$, belongs to $C_{i}\left(p^{*}\right)$, is in the interior of $B_{i}\left(p^{*}\right)$, and is also in the interior of $B_{i}\left(p^{j}\right)$, for high $j$ :

$$
U_{i}\left(x_{i}^{\prime}\right)>U_{i}\left(x_{i}^{*}\right) ; \quad x_{i}^{\prime} \in C_{i}\left(p^{*}\right) ; \quad p^{*} \cdot x_{i}^{\prime}<p^{*} \cdot e_{i} ; \quad p^{j} \cdot x_{i}^{\prime}<p^{j} \cdot e_{i}, \forall j \geq j_{0} .
$$

Again, by continuity of $U_{i}$, there exists $\epsilon>0$ such that $d\left(x_{i}, x_{i}^{\prime}\right)<\epsilon$ implies that $U_{i}\left(x_{i}\right)>U_{i}\left(x_{i}^{*}\right)$, with $x_{i}$ in the interior of $B_{i}\left(p^{*}\right)$. For $j \geq j_{1} \geq j_{0}, U_{i}\left(x_{i}\right)>U_{i}\left(x_{i}^{j}\right)$ (notice that we are considering $U_{i}$ and not $\left.U_{i}^{j}\right)$ and $x_{i}$ is in the interior of $B_{i}\left(p^{j}\right)$.

Let $j_{2} \geq j_{1}$ be sufficiently high for $d\left(p^{j}, p^{*}\right)<\epsilon, \forall j \geq j_{2}$.

Consider, for easiness of exposition and without loss of generality, the following element of the agent's information partition: $P_{i}(s)=\{1, \ldots, s\}$. It should be clear that this reasoning extends to any element of $P_{i}$. Since $x_{i}^{\prime} \in C_{i}\left(p^{*}\right)$, the deliverability conditions are satisfied: 


$$
\left\{\begin{array} { l } 
{ p ^ { * 1 } \cdot x _ { i } ^ { \prime 1 } \leq p ^ { * 1 } \cdot x _ { i } ^ { \prime 2 } ; } \\
{ \cdots } \\
{ p ^ { * 1 } \cdot x _ { i } ^ { \prime 1 } \leq p ^ { * 1 } \cdot x _ { i } ^ { \prime s } ; } \\
{ p ^ { * 2 } \cdot x _ { i } ^ { \prime 2 } \leq p ^ { * 2 } \cdot x _ { i } ^ { \prime 1 } ; } \\
{ \cdots } \\
{ p ^ { * 2 } \cdot x _ { i } ^ { \prime 2 } \leq p ^ { * 2 } \cdot x _ { i } ^ { \prime s } ; } \\
{ \cdots } \\
{ \cdots } \\
{ p ^ { * s } \cdot x _ { i } ^ { \prime s } \leq p ^ { * s } \cdot x _ { i } ^ { \prime 1 } ; } \\
{ \cdots } \\
{ p ^ { * s } \cdot x _ { i } ^ { \prime s } \leq p ^ { * s } \cdot x _ { i } ^ { \prime s - 1 } . }
\end{array} \Leftrightarrow \left\{\begin{array}{l}
p^{* 1} \cdot x_{i}^{\prime 2}-p^{* 1} \cdot x_{i}^{\prime 1}=k^{12} \geq 0 ; \\
\cdots \\
p^{* 1} \cdot x_{i}^{\prime s}-p^{* 1} \cdot x_{i}^{\prime 1}=k^{1 s} \geq 0 ; \\
p^{* 2} \cdot x_{i}^{\prime 1}-p^{* 2} \cdot x_{i}^{\prime 2}=k^{21} \geq 0 ; \\
\cdots \\
p^{* 2} \cdot x_{i}^{\prime s}-p^{* 2} \cdot x_{i}^{\prime 2}=k^{2 s} \geq 0 ; \\
\cdots \\
\cdots \\
p^{* s} \cdot x_{i}^{\prime 1}-p^{* s} \cdot x_{i}^{\prime s}=k^{s 1} \geq 0 ; \\
\cdots \\
p^{* s} \cdot x_{i}^{\prime s-1}-p^{* s} \cdot x_{i}^{\prime s}=k^{s, s-1} \geq 0 .
\end{array}\right.\right.
$$

We will find an $x_{i}$ that is a neighbor of $x_{i}^{\prime}$ and belongs to $C_{i}\left(p^{j}\right)$ (contradicting the fact that $x_{i}^{j}$ is individually optimal at prices $\left.p^{j}\right)$. This proves (4) by contradiction.

Case 1: All inequalities are such that $k^{s t}>0$.

With $x_{i}$ such that $d\left(x_{i}, x_{i}^{\prime}\right)<\epsilon$, denote $d x_{i}=x_{i}-x_{i}^{\prime}$ and $d p^{j}=p^{j}-p^{*}$. Manipulating a deliverability condition:

$$
\begin{aligned}
& p^{* s} \cdot x_{i}^{\prime t}-p^{* s} \cdot x_{i}^{\prime s}=\left(p^{j s}-d p^{j s}\right) \cdot\left(x_{i}^{t}-d x_{i}^{t}\right)-\left(p^{j s}-d p^{j s}\right) \cdot\left(x_{i}^{s}-d x_{i}^{s}\right)=k^{s t} \Leftrightarrow \\
& \Leftrightarrow p^{j s} \cdot x_{i}^{t}-p^{j s} \cdot x_{i}^{s}=k^{s t}+p^{j s} \cdot d x_{i}^{t}+d p^{s} \cdot\left(x_{i}^{t}-d x_{i}^{t}\right)-p^{j s} \cdot d x_{i}^{s}-d p^{s} \cdot\left(x_{i}^{s}-d x_{i}^{s}\right) \Leftrightarrow \\
& \Leftrightarrow p^{j s} \cdot x_{i}^{t}-p^{j s} \cdot x_{i}^{s}>k^{s t}-\epsilon-\epsilon\left(\left\|e_{T}\right\|+\epsilon\right)-\epsilon-\epsilon\left(\left\|e_{T}\right\|+\epsilon\right) \Leftrightarrow \\
& \Leftrightarrow p^{j s} \cdot x_{i}^{t}-p^{j s} \cdot x_{i}^{s}>k^{s t}-2 \epsilon\left(\left\|e_{T}\right\|+1-\epsilon\right) .
\end{aligned}
$$

Let $k^{\text {min }}=\min _{t \in P_{i}(s)} k^{s t}$. Choose a smaller $\epsilon>0$, if necessary, to make $2 \epsilon\left(\left\|e_{T}\right\|+1-\epsilon\right)<k^{m i n}$. This guarantees that the strict inequalities for $x_{i}^{\prime}$ and $p^{*}$ remain strict for any $x_{i}$ s.t. $d\left(x_{i}, x_{i}^{\prime}\right)<\epsilon$, under $p^{j}$ with $j \geq j_{2}$.

There is no utility penalty, therefore, $U_{i}^{j}\left(x_{i}\right)>U_{i}^{j}\left(x_{i}^{j}\right)$. Contradiction. The consumption plan in the equilibrium sequence, $x_{i}^{j}$, is not a maximizer of $U_{i}^{j}$.

Case 2: For every $t \in P_{i}(s)$, prices $p^{* s}$ and $p^{* t}$ are not parallel.

The difference relative to Case 1 lies in checking that the inequalities which are not strict at $\left(x_{i}^{\prime}, p^{*}\right)$ are still satisfied at $\left(x_{i}, p^{j}\right)$, for high $j$. The inequalities that are not strict are those for which $k^{s t}=0$.

Let $\gamma^{s t}=\left(1-\frac{p^{* s} \cdot p^{* t}}{\left\|p^{* s}\right\|\left\|p^{* t}\right\|}\right)\left\|p^{* s}\right\|$ and $\gamma^{\min }=\min _{t \in P_{i}(s)} \gamma^{s t}>0$. 
Define $k^{\text {min }}$ as the minimum among the strictly positive $k^{s t}$ and (as in Case 1) choose $\epsilon>0$ such that $2 \epsilon\left(\left\|e_{T}\right\|+1-\epsilon\right)<k^{\min }$, to preserve the strict inequalities.

Select displacements from $x_{i}^{\prime}$ to $x_{i}$ that are parallel to $p^{*}$, choosing $x_{i}$ such that: $d x_{i}^{s}=-\frac{\epsilon}{2} \frac{p^{* s}}{\left\|p^{* s}\right\|}$.

Let $\epsilon_{2}=\frac{\epsilon \gamma^{\min }}{8\left\|e_{T}\right\|}$, and consider $j_{3} \geq j_{2}$ that is high enough for: $d\left(p^{j}, p^{*}\right)<\min \left\{\epsilon_{2}, \epsilon\right\}, \forall j \geq j_{3}$.

Consider an inequality that is not strict for $p^{*}$ and $x_{i}^{\prime}\left(k^{a b}=0\right)$. Let's verify that this deliverability condition still holds for $p^{j}$ and $x_{i}$ :

$$
\begin{aligned}
& p^{j a} \cdot x_{i}^{b}-p^{j a} \cdot x_{i}^{a}=\left(p^{* a}+d p^{j a}\right) \cdot\left(x_{i}^{\prime b}+d x_{i}^{b}\right)-\left(p^{* a}+d p^{j a}\right) \cdot\left(x_{i}^{\prime a}+d x_{i}^{a}\right)= \\
& =p^{* a} \cdot\left(x_{i}^{\prime b}+d x_{i}^{b}\right)+d p^{j a} \cdot\left(x_{i}^{\prime b}+d x_{i}^{b}\right)-p^{* a} \cdot\left(x_{i}^{\prime a}+d x_{i}^{a}\right)-d p^{j a} \cdot\left(x_{i}^{\prime a}+d x_{i}^{a}\right)= \\
& =p^{* a} \cdot d x_{i}^{b}+d p^{j a} \cdot\left(x_{i}^{\prime b}+d x_{i}^{b}\right)-p^{* a} \cdot d x_{i}^{a}-d p^{j a} \cdot\left(x_{i}^{\prime a}+d x_{i}^{a}\right)> \\
& >p^{* a} \cdot d x_{i}^{b}-\epsilon_{2}\left(\left\|e_{T}\right\|+\epsilon\right)-p^{* a} \cdot d x_{i}^{a}-\epsilon_{2}\left(\left\|e_{T}\right\|+\epsilon\right)= \\
& =p^{* a} \cdot d x_{i}^{b}-p^{* a} \cdot d x_{i}^{a}-2 \epsilon_{2}\left(\left\|e_{T}\right\|+\epsilon\right)> \\
& >-p^{* a} \cdot \frac{\epsilon}{2} \frac{p^{* b}}{\left\|p^{* b}\right\|}+p^{* a} \cdot \frac{\epsilon}{2} \frac{p^{* a}}{\left\|p^{* a}\right\|}-4 \epsilon_{2}\left\|e_{T}\right\|= \\
& =\frac{\epsilon}{2} \frac{p^{* a} \cdot p^{* a}}{\left\|p^{* a}\right\|\left\|p^{* a}\right\|}\left\|p^{* a}\right\|-\frac{\epsilon}{2} \frac{p^{* a} \cdot p^{* b}}{\left\|p^{* a}\right\|\left\|p^{* b}\right\|}\left\|p^{* a}\right\|-\frac{\epsilon}{2} \gamma^{m i n}= \\
& =\frac{\epsilon}{2} \gamma^{a b}-\frac{\epsilon}{2} \gamma^{m i n} \geq 0
\end{aligned}
$$

In sum: $p^{j a} \cdot x_{i}^{b}-p^{j a} \cdot x_{i}^{a}>0$. The deliverability condition is verified, and thus $U_{i}^{j}\left(x_{i}\right)>U_{i}^{j}\left(x_{i}^{j}\right)$. Contradiction.

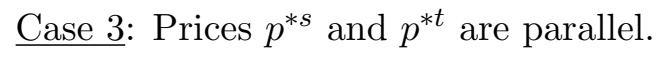

The same displacement as in Case 2, $d x_{i}^{s}=-\frac{\epsilon}{2} \frac{p^{* s}}{\left\|p^{* s}\right\|}$, is good for the case in which prices $p^{* a}$ and $p^{* b}$ are parallel. In this case: $x_{i}^{\prime a}=x_{i}^{\prime b}$ and also $d x_{i}^{a}=d x_{i}^{b}$. Hence, $x_{i}^{a}=x_{i}^{b}$ and the conditions remain satisfied in equality.

All deliverability conditions are satisfied, therefore: $U_{i}^{j}\left(x_{i}\right)=U_{i}\left(x_{i}\right)>U_{i}\left(x_{i}^{j}\right) \geq U_{i}^{j}\left(x_{i}^{j}\right)$. The consumption plan $x_{i}^{j}$ does not maximize $U_{i}^{j}$, because $x_{i}$ is preferred. This contradiction proves $(4)$.

\section{Proof of Theorem 2:}

Suppose, by way of contradiction, that: (i) all the agents $i \in G$ prefer to announce state $t$ when the true state is $s, u_{i}^{s}\left(e_{i}^{s}+x_{i}^{t *}-e_{i}^{t}\right)>u_{i}^{s}\left(x_{i}^{s *}\right), \forall i \in G$; (ii) that the agents in $G$ agree that they should deviate, $P_{i}(s)=P_{j}(s), \forall(i, j) \in G^{2}$; and (iii) that the false report is not incompatible with the truthful 
reports of the other agents, $t \in \cap_{j \in \mathcal{I} \backslash G} P_{j}(s)$.

Since any state can be verified by at least one agent, there is some agent $i \in G$ for who $P_{i}(s)=\{s\}$. Therefore, from hypothesis (ii), $P_{j}(s)=\{s\}, \forall j \in G$.

Denote by $z_{j}^{s *}$ and $z_{j}^{t *}$ the net trades of each agent $j$ in states $s$ and $t$, respectively. The deliverability constraints imply that, for all $j \in \mathcal{I} \backslash G: p^{s} \cdot z_{j}^{s *} \leq p^{s} \cdot z_{j}^{t *}$.

Denote by $Z_{G}^{s *}$ and $Z_{-G}^{s *}$ the aggregate net trade in state $s$ of the agents inside and outside the coalition $G$, respectively. Adding the deliverability constraints of the agents outside $G$, we obtain: $p^{s} \cdot Z_{-G}^{s *} \leq p^{s} \cdot Z_{-G}^{t *}$.

Since equilibrium prices are positive (see Lemma 3) and any state can be verified by at least one agent, we have exact feasibility. Therefore: $Z_{G}^{s *}=-Z_{-G}^{s *}$ and $Z_{G}^{t *}=-Z_{-G}^{t *}$.

This means that $p^{s} \cdot Z_{G}^{s *} \geq p^{s} \cdot Z_{G}^{t *}$, which implies that $\exists i \in G, p^{s} \cdot z_{i}^{s *} \geq p^{s} \cdot z_{i}^{t *}$.

But then we have a contradiction with the original choice of $z_{i}^{s *}$ instead of $z_{i}^{t *}$ to be the net trade in state $s$, because $z_{i}^{t *}$ at least as cheap and strictly preferred to $z_{i}^{s *}$. Since the choice of agent $i$ for delivery in state $s$ was not subject to any deliverability constraint (because $P_{i}(s)=\{s\}$ ), there cannot exist a net trade $z_{i}^{t *}$ that is at least as cheap and strictly preferred to $z_{i}^{s *}$.

\section{Appendix 2: Example of non-existence of equilibrium}

Consider an economy in which two agents trade a single good under uncertainty. There are three states of nature, and the endowments depend on the state of nature:

$e_{A}=(100,100,1)$ and $e_{B}=(1,100,100)$.

Agents only observe their endowments:

$$
P_{A}=\{\{1,2\} ;\{3\}\} \text { and } P_{B}=\{\{1\} ;\{2,3\}\} .
$$

The different states occur with objective and publicly known probabilities:

$$
\mu=\left(\mu^{1}, \mu^{2}, \mu^{3}\right)=(0.45,0.1,0.45)
$$

Risk aversion induces agents to trade ex ante, in order to maximize expected utility:

$$
U_{i}\left(x_{i}\right)=\sum_{s=1}^{S} \mu^{s} \sqrt{x_{i}^{s}} .
$$

Prices in states 1 and 3 must be strictly positive, or else the demands of agent $B$ and $A$ would be 
infinite for the corresponding contingent goods.

With strictly positive prices for all the contingent goods, if agents selected different consumption levels in states that they did not distinguish, then they would end up receiving the cheapest of the alternatives, which would be the one with the lowest consumption level. In this case, we must have:

$$
x_{A}=\left(x_{A}^{12}, x_{A}^{12}, x_{A}^{3}\right) \text { and } x_{B}=\left(x_{B}^{1}, x_{B}^{23}, x_{B}^{23}\right) .
$$

Since agents are at the frontier of their budget sets:

$$
\left\{\begin{array}{l}
\left(p^{1}+p^{2}\right) x_{A}^{12}+p^{3} x_{A}^{3}=100\left(p^{1}+p^{2}\right)+p^{3} \\
p^{1} x_{B}^{1}+\left(p^{2}+p^{3}\right) x_{B}^{23}=p^{1}+100\left(p^{2}+p^{3}\right) .
\end{array}\right.
$$

Adding the two:

$$
p^{1}\left(x_{A}^{12}+x_{B}^{1}\right)+p^{2}\left(x_{A}^{12}+x_{B}^{23}\right)+p^{3}\left(x_{A}^{3}+x_{B}^{23}\right)=101 p^{1}+200 p^{2}+101 p^{3} .
$$

For this to be an equilibrium, the allocation must be feasible:

$$
\left\{\begin{array}{l}
x_{A}^{12}+x_{B}^{1} \leq 101 \\
x_{A}^{12}+x_{B}^{23} \leq 200 \\
x_{A}^{3}+x_{B}^{23} \leq 101
\end{array}\right.
$$

With strictly positive prices, the conditions are verified in equality. This implies that the allocation is of the form:

$$
\left\{\begin{array}{l}
x_{A}=\left(x_{A}^{12}, x_{A}^{12}, x_{A}^{3}\right)=\left(x_{A}^{3}+99, x_{A}^{3}+99, x_{A}^{3}\right) \\
x_{B}=\left(x_{B}^{1}, x_{B}^{23}, x_{B}^{23}\right)=\left(x_{B}^{1}, x_{B}^{1}+99, x_{B}^{1}+99\right) .
\end{array}\right.
$$

The only individually rational allocation of this form corresponds to the initial endowments. There is no trade. But are agents maximizing their utility levels?

$$
\left\{\begin{array} { l } 
{ x _ { A } = ( 1 0 0 , 1 0 0 , 1 ) ; } \\
{ x _ { B } = ( 1 , 1 0 0 , 1 0 0 ) . }
\end{array} \Rightarrow \left\{\begin{array}{l}
U\left(x_{A}\right)=0.45 * 10+0.1 * 10+0.45 * 1=5.95 \\
U\left(x_{B}\right)=0.45 * 1+0.1 * 10+0.45 * 10=5.95 .
\end{array}\right.\right.
$$

Suppose that $p^{1}=p^{3}$. Agent $A$ can trade consumption in state 1 for consumption in state 3 . But consuming less in state 1 implies that delivery in state 2 will also be of this lower quantity. In any case, the agent can select:

$$
x_{1}^{\prime}=\left(x_{1}^{\prime 12}, x_{1}^{\prime 12}, x_{1}^{\prime 3}\right)=(81,81,20) .
$$

The corresponding utility level is:

$$
U\left(x_{1}^{\prime}\right)=0.45 * 9+0.1 * 9+0.45 * 4.47=6.96 .
$$

In the case with asymmetric prices $\left(p^{1} \neq p^{3}\right)$, the same trade is even more favorable for one of the 
agents. We reached a contradiction, implying that there is no equilibrium with strictly positive prices. With $p^{2}=0$, an alternative bundle can be big enough to violate feasibility and still be deliverable. The deliverability restriction is not relevant because it is of the form $0 \cdot x^{2} \leq 0 \cdot x^{s}$. Agents can choose a consumption level for state 2 that is big enough to violate feasibility and still desire to increase it. There cannot be a rational expectations equilibrium with $p^{2}=0$.

\section{Appendix 3: The deliverability correspondence}

The set of bundles that satisfy the deliverability restrictions depends on the prevailing prices. Consider the correspondence from prices to the set of deliverable bundles:

$$
\begin{aligned}
& C_{i}: \Delta^{S L} \rightarrow \mathbb{R}_{+}^{S L} ; \\
& C_{i}(p)=\left\{x \in \mathbb{R}_{+}^{S L}: \forall s \in \mathcal{S}, p^{s} \cdot x^{s}=\min _{t \in P_{i}(s)}\left\{p^{s} \cdot x^{t}\right\}\right\} .
\end{aligned}
$$

If the correspondence $B_{i}(p) \cap C_{i}(p)$ were continuous, we could apply Berge's maximum theorem and Kakutani's fixed point theorem to establish existence of equilibrium in economies with uncertain delivery.

Upper hemicontinuity of $C_{i}$ at $p_{0}$ means that, given an arbitrary open set, $V$, containing $C_{i}\left(p_{0}\right)$, there exists $\delta>0$ such that for all $p \in B\left(p_{0}, \delta\right)$, we have $C_{i}(p) \subseteq V$.

The correspondence is closed since all the restrictions are inequalities which are not strict. With a compact range, a closed-valued correspondence is upper hemicontinuous if and only if it is closed. Therefore, when restricted to a bounded economy (for example, by the total initial endowments in the economy), $C_{i}$ is upper hemicontinuous.

Lower hemicontinuity of $C_{i}$ at $p_{0}$ means that given an arbitrary open set, $V$, intersecting $C_{i}\left(p_{0}\right)$, there exists $\delta>0$ such that for all $p \in B\left(p_{0}, \delta\right)$, the image $C_{i}(p)$ also intersects $V$.

The correspondence under study, $C_{i}$, is not lower hemicontinuous. Lower hemicontinuity fails when prices in are null $\left(p^{s}=0\right)$ or collinear $\left(p^{s}=a p^{t}\right)$.

When prices are null, the deliverability restrictions disappear. It is always true that $0 \cdot x^{s} \leq 0 \cdot x^{t}$. But with a small perturbation, the restrictions appear. This is why l.h.c. fails.

When prices are collinear, the failure of l.h.c. is more subtle.

Consider an economy with two goods, $A$ and $B$, and two states of nature, $s$ and $t$. Let $p_{0}=\left(p_{0}^{s}, p_{0}^{t}\right)=$ $\left(p_{0}^{A s}, p_{0}^{B s} ; p_{0}^{A t}, p_{0}^{B t}\right)=\left(\frac{1}{4}, \frac{1}{4} ; \frac{1}{4}, \frac{1}{4}\right)$. The bundle $x_{0}=(1,0 ; 0,1)$ belongs to the deliverable set, since: 


$$
\begin{aligned}
& p_{0}^{s} \cdot x_{0}^{s} \leq p_{0}^{s} \cdot x_{0}^{t} \Leftrightarrow \frac{1}{4} \leq \frac{1}{4}, \text { and } \\
& p_{0}^{t} \cdot x_{0}^{t} \leq p_{0}^{t} \cdot x_{0}^{s} \Leftrightarrow \frac{1}{4} \leq \frac{1}{4} .
\end{aligned}
$$

Delivering $(1,0)$ in state $s$ and $(0,1)$ in state $t$ does not violate deliverability because both bundles have the same price in both states.

A small perturbation in prices can make $(0,1)$ cheaper in state $s$ and $(1,0)$ cheaper in state $t$. Consider an open ball around $x_{0}$ with radius $0<\epsilon<\frac{1}{10}$. After a perturbation in prices to $p=\left(\frac{1}{4}+\delta, \frac{1}{4}-\delta, \frac{1}{4}-\right.$ $\left.\delta, \frac{1}{4}+\delta\right)$, this ball does not intersect the deliverable set.

Suppose that there existed a vector $d x=\left(\epsilon^{A s}, \epsilon^{B s}, \epsilon^{A t}, \epsilon^{B t}\right)$ such that $x=\left(1+\epsilon^{A s}, \epsilon^{B s} ; \epsilon^{A t}, 1+\epsilon^{B t}\right)$ is inside that open ball and belongs to the deliverable set:

$$
\begin{aligned}
& \text { (i) } \quad\left(\frac{1}{4}+\delta, \frac{1}{4}-\delta\right) \cdot\left(1+\epsilon^{A s}, \epsilon^{B s}\right) \leq\left(\frac{1}{4}+\delta, \frac{1}{4}-\delta\right) \cdot\left(\epsilon^{A t}, 1+\epsilon^{B t}\right) \Leftrightarrow \\
& \Leftrightarrow\left(\frac{1}{4}+\delta\right)\left(1+\epsilon^{A s}\right)+\left(\frac{1}{4}-\delta\right) \epsilon^{B s} \leq\left(\frac{1}{4}+\delta\right) \epsilon^{A t}+\left(\frac{1}{4}-\delta,\right)\left(1+\epsilon^{B t}\right) \Leftrightarrow \\
& \Leftrightarrow \frac{1}{4}+\frac{1}{4} \epsilon^{A s}+\delta+\delta \epsilon^{A s}+\frac{1}{4} \epsilon^{B s}-\delta \epsilon^{B s} \leq \frac{1}{4} \epsilon^{A t}+\delta \epsilon^{A t}+\frac{1}{4}+\frac{1}{4} \epsilon^{B t}-\delta-\delta \epsilon^{B t} \Leftrightarrow \\
& \Leftrightarrow \frac{1}{4}\left(\epsilon^{A s}+\epsilon^{B s}-\epsilon^{A t}-\epsilon^{B t}\right)+\delta\left(\epsilon^{A s}-\epsilon^{B s}-\epsilon^{A t}+\epsilon^{B t}\right) \leq-2 \delta ; \\
& (i i) \quad\left(\frac{1}{4}-\delta, \frac{1}{4}+\delta\right) \cdot\left(\epsilon^{A t}, 1+\epsilon^{B t}\right) \leq\left(\frac{1}{4}-\delta, \frac{1}{4}+\delta\right) \cdot\left(1+\epsilon^{A s}, \epsilon^{B s}\right) \Leftrightarrow \\
& \Leftrightarrow\left(\frac{1}{4}-\delta\right) \epsilon^{A t}+\left(\frac{1}{4}+\delta\right)\left(1+\epsilon^{B t}\right) \leq\left(\frac{1}{4}-\delta\right)\left(1+\epsilon^{A s}+\left(\frac{1}{4}+\delta\right) \epsilon^{B s}\right) \Leftrightarrow \\
& \Leftrightarrow \frac{1}{4}\left(\epsilon^{A t}+1+\epsilon^{B t}-1-\epsilon^{A s}-\epsilon^{B s}\right)+\delta\left(-\epsilon^{A t}+1+1+\epsilon^{B t}+\epsilon^{A s}-\epsilon^{B s}\right) \leq 0 \Leftrightarrow \\
& \Leftrightarrow \frac{1}{4}\left(\epsilon^{A t}+\epsilon^{B t}-\epsilon^{A s}-\epsilon^{B s}\right)+\delta\left(-\epsilon^{A t}+\epsilon^{B t}+\epsilon^{A s}-\epsilon^{B s}\right) \leq-2 \delta .
\end{aligned}
$$

Adding the two inequalities, we obtain:

$$
\delta\left(\epsilon^{A s}-\epsilon^{B s}-\epsilon^{A t}+\epsilon^{B t}\right) \leq-2 \delta \Leftrightarrow \epsilon^{A s}-\epsilon^{B s}-\epsilon^{A t}+\epsilon^{B t} \leq-2 .
$$

Which is impossible, because $\epsilon^{A s}-\epsilon^{B s}-\epsilon^{A t}+\epsilon^{B t} \geq-4 \epsilon>-\frac{4}{10}$.

\section{References}

Aliprantis, C.D. and K. Border, (2007), "Infinite Dimensional Analysis", 3rd ed., Springer.

Allen, B. (1981), "Generic Existence of Completely Revealing Equilibria for Economies with Uncertainty when Prices Convey Information", Econometrica, 49 (5), pp. 1173-1199.

Angeloni, L. and Martins-da-Rocha, V.F. (2009), "Large economies with differential information and 
without free disposal", Economic Theory, 38 (2), pp. 263-286.

Arrow, K.J. (1953), "The Role of Securities in the Optimal Allocation of Risk-Bearing", Econometrie, translated and reprinted in 1964, Review of Economic Studies, 31 (2), pp. 91-96.

Arrow, K.J. and G. Debreu (1954), "Existence of an Equilibrium for a Competitive Economy", Econometrica, 22 (3), pp. 265-290.

Arrow, K.J. and F. Hahn (1971), "General Competitive Analysis", Holden Day, San Francisco.

Correia-da-Silva, J. and C. Hervés-Beloso (2008), "Subjective Expectations Equilibrium in Economies with Uncertain Delivery", Journal of Mathematical Economics, 44 (7-8), pp. 641-650.

Correia-da-Silva, J. and C. Hervés-Beloso (2009), "Prudent Expectations Equilibrium in Economies with Uncertain Delivery", Economic Theory, 39 (1), pp. 67-92.

Debreu, G. (1959), "Theory of Value", Wiley, New York.

Einy, E., D. Moreno and B. Shitovitz (2001), "Competitive and core allocations in large economies with differential information", Economic Theory, 18 (2), pp. 321-332.

Glycopantis D., A. Muir and N.C. Yannelis (2003), "On extensive form implementation of contracts in differential information economies", Economic Theory, 21 (2-3), pp. 495-526.

Glycopantis, D. and N.C. Yannelis (2005), "Differential Information Economies", Studies in Economic Theory, 19, Springer, New York.

Hervés-Beloso, C., Moreno-García, E. and Yannelis, N.C. (2005), "Characterization and Incentive Compatibility of Walrasian Expectations Equilibrium in Infinite Dimensional Commodity Spaces", Economic Theory, 26 (2), pp. 361-381.

Hervés-Beloso C., V.F. Martins-da-Rocha and P.K. Monteiro (2009), "Equilibrium theory with asymmetric information and infinitely many states", Economic Theory, 38 (2), pp. 295-320.

Koutsougeras, L.C. and N.C. Yannelis (1993), "Incentive compatibility and information superiority of the core of an economy with differential information", Economic Theory, 3 (2), pp. 195-216.

Krasa, S. and N.C. Yannelis (1994), "The value allocation of an economy with differential information", Econometrica, 62 (4), pp. 881-900.

McKenzie, L.W. (1954), "On Equilibrium in Graham's Model of World Trade and Other Competitive Systems", Econometrica, 22 (2), pp. 147-161.

Muth, J.F. (1961), "Rational Expectations and the Theory of Price Movements", Econometrica, 29 (3), 
pp. 315-335.

Podczeck, K. and N.C. Yannelis (2008), "Equilibrium theory with asymmetric information and with infinitely many commodities", Journal of Economic Theory, 141 (1), pp. 152-183.

Radner, R. (1968), "Competitive Equilibrium Under Uncertainty", Econometrica, 36 (1), pp. 31-58.

Radner, R. (1979), "Rational Expectations Equilibrium: Generic Existence and the Information Revealed by Prices", Econometrica, 47 (3), pp. 655-678.

Yannelis, N.C. (1991), "The Core of an Economy with Differential Information", Economic Theory, 1 (2), pp. 183-197. 